\title{
Nonperturbative 3D Lorentzian quantum gravity
}

\author{
J. Ambjørn* \\ The Niels Bohr Institute, Blegdamsvej 17, DK-2100 Copenhagen $\varnothing$, Denmark \\ J. Jurkiewicz ${ }^{\dagger}$ \\ Institute of Physics, Jagellonian University, Reymonta 4, PL 30-059 Krakow, Poland \\ R. Loll \\ Albert-Einstein-Institut, Max-Planck-Institut für Gravitationsphysik, Am Mühlenberg 1, D-14476 Golm, Germany
}

(Received 8 December 2000; published 24 July 2001)

\begin{abstract}
We have recently introduced a discrete model of Lorentzian quantum gravity, given as a regularized nonperturbative state sum over simplicial Lorentzian space-times, each possessing a unique Wick rotation to the Euclidean signature. We investigate here the phase structure of the Wick-rotated path integral in three dimensions with the aid of computer simulations. After fine tuning the cosmological constant to its critical value, we find a whole range of the gravitational coupling constant $k_{0}$ for which the functional integral is dominated by nondegenerate three-dimensional space-times. We therefore have a situation in which a well-defined ground state of extended geometry is generated dynamically from a nonperturbative state sum of fluctuating geometries. Remarkably, its macroscopic scaling properties resemble those of a semiclassical spherical universe. Measurements so far indicate that $k_{0}$ defines an overall scale in this extended phase, without affecting the physics of the continuum limit. These findings provide further evidence that discrete Lorentzian gravity is a promising candidate for a nontrivial theory of quantum gravity.
\end{abstract}

DOI: 10.1103/PhysRevD.64.044011 PACS number(s): 04.60.Gw, 04.20.Gz, 04.60.Kz, 04.60.Nc

\section{INTRODUCTION}

The aim of discrete approaches to quantum gravity is the nonperturbative construction of a quantum theory of gravity as the continuum limit of a discretized state sum, analogous to Feynman's construction of the quantum-mechanical propagator of a particle [1]. Among quantizers of gravity, path-integral formulations have fallen into disrepute, both because of the nonrenormalizability of the perturbation series and because of the unboundedness of the action, which seems to render Euclidean approaches (at least formal continuum path integrals and covariantly formulated cosmological models) ill defined. Although this does not necessarily constitute an obstacle to the existence of a nonperturbative path integral, it raises the question of how such a quantity is to be constructed.

There is little hope of evaluating the continuum path integral directly, because of the complicated functional form of the gravitational action and because of quantum-field theoretic divergences, with the ensuing need to regularize in a way compatible with the symmetries of the theory. This leads to additional complications, since the invariance group of general relativity is the group of space-time diffeomorphisms, and not the Poincare group of quantum field theory on a fixed, flat background, so that standard regularization methods cannot be applied.

There are alternative regularization schemes, much in the spirit of Feynman's treatment of the nonrelativistic particle,

\footnotetext{
*Electronic address: ambjorn@nbi.dk

†Electronic address: jurkiewi@thrisc.if.uj.edu.pl

†Electronic address: loll@aei-potsdam.mpg.de
}

formulated on discretized versions of the space of all spacetime geometries (that is, of space-time metrics modulo diffeomorphisms). One popular class of models is based on applying Regge's idea [2] of approximating smooth spacetimes $\left(M, g_{\mu \nu}\right)$ by piecewise linear, simplicial manifolds in a quantized context. Unfortunately such models have to date produced little convincing evidence of interesting continuum physics, for reasons that are ultimately not well understood [see [3] for a recent review of discrete models in four dimensions (4D)].

A serious criticism one can level at these models is that they are all formulated for positive-definite Euclidean (Riemannian) metrics $g_{\mu \nu}^{\mathrm{Eu}}$, and not for physical metrics $g_{\mu \nu}^{\mathrm{Lor}}$ of indefinite, Lorentzian signature. This is done for technical reasons, since in a concrete regularized formulation one must make sure that the state sum/integral $Z$ converges. However, it is important to realize that, in a nonperturbative context,

$$
\begin{aligned}
Z^{\mathrm{Lor}}= & \int_{\operatorname{Lor}(M) / \operatorname{Diff}(M)}\left[\mathcal{D} g_{\mu \nu}^{\mathrm{Lor}}\right] e^{i S\left[g^{\mathrm{Lor}}\right]} \\
& \mapsto Z^{\mathrm{Eu}}=\int_{\operatorname{Eu}(M) / \operatorname{Diff}(M)}\left[\mathcal{D} g_{\mu \nu}^{\mathrm{Eu}}\right] e^{-S\left[g^{\mathrm{Eu}}\right]}
\end{aligned}
$$

is an ad hoc substitution: away from a handful of metrics with special symmetries (for example, flat Minkowski space), there is no straightforward "Wick rotation" $g_{\mu \nu}^{\text {Lor }} \mapsto g_{\mu \nu}^{\mathrm{Eu}}$ (or, equivalently, $t \mapsto-i t$ ).

It may well be that the absence of an interesting continuum limit in these statistical models of dynamical geometries is related to the absence of any Lorentzian structure in their partition functions. This observation has motivated us to construct a well-defined discrete quantum gravity model 
in terms of Lorentzian geometries. A suitable starting point is the method of dynamical triangulations, a variant of the quantum Regge calculus program, which has the advantage of being amenable to both numerical simulations and analytic treatments. Following Regge's original concept of describing "geometry without coordinates," the "sum over all paths" is performed directly over physically inequivalent geometries. Unlike in continuum path-integral approaches, there is no need to introduce coordinates and to subsequently gauge-fix them. (In this sense, diffeomorphism invariance is manifest.) In our model, instead of using equilateral Euclidean triangulations, we take the state sum over a certain class of Lorentzian Regge manifolds, obtained by gluing together a number of simple simplicial Lorentzian building blocks.

The model has been constructed explicitly in two, three, and four space-time dimensions [4-6], and been solved exactly in $d=2[4,7]$. Each Lorentzian geometry (or "history") has a foliated structure, with a (discrete) proper time $t$ labeling successive spatial slices. (Note that this proper time is simply one of the parameters characterizing the discrete geometries, and not a "gauge choice," since the formalism is completely coordinate invariant from the outset.) In addition, each history has a causal structure, induced from the piecewise linear Lorentzian metric structure. Each spatial slice is a $(d-1)$-dimensional equilateral triangulation of Euclidean simplices, with squared edge lengths $l_{\text {space }}^{2}=a^{2}$, and spatial topology changes are not allowed (in line with the continuum notion of causality).

A unique Wick rotation is defined on every Lorentzian history. It maps a given triangulation with certain assignments of edge lengths into the same topological triangulation, but with the (squared) edge lengths of its time-like links (which interpolate between the spatial slices) redefined from $l_{\text {time }}^{2}=-\alpha a^{2}$ to $l_{\text {time }}^{2}=+\alpha a^{2}$, where $\alpha>0$. This leads to an analytic continuation from Lorentzian to Euclidean signature of the Regge action in the complex $\alpha$ plane and gives a precise meaning to the map Eq. (1).

For finite (discrete) volume, the Lorentzian gravity models thus obtained are well defined in the sense of being statistical systems whose transfer matrix is bounded and positive. One is then interested in whether they exhibit any critical behavior as functions of the bare coupling constants, leading to continuum theories of quantum gravity. Second, one wants to compare their properties with those of the corresponding Euclidean dynamically triangulated models. It should perhaps be emphasized at this point that our nonperturbative path-integral method is in principle exact (and not formal). It does involve a specific choice of a "measure," but one would expect from universality arguments that the fine details of any choices made at the discretized level will not alter the continuum theory.

As already mentioned above, in $d=2$ the Lorentzian model is exactly soluble and lies in a different universality class from its Euclidean counterpart (which can also be solved exactly and is better known as 2D Liouville quantum gravity). Its geometric properties are different, which also affects its behavior when matter is coupled to the gravitational degrees of freedom [8,9]. Quite remarkably, and unlike Liouville gravity, the coupled system remains well behaved beyond the so-called " $c=1$ barrier" (in our case, this is equivalent to the number of coupled Ising models exceeding 2).

These are very interesting results from the point of view of systems of two-dimensional random geometries, but our ultimate interest lies in the physical, four-dimensional case, and the physics of "general relativity" in two, three, and four dimensions is very different. Where dynamically triangulated Euclidean models seem to go wrong in $d>2$ is in the dominance of highly degenerate geometries over their statistical ensembles. It is encouraging that one can show the absence of the same type of geometries from the Lorentzian ensemble $[5,6]$, but one could still be worried about the occurrence of (less extreme) pathologies. The only way to determine whether Lorentzian gravity does indeed solve the problems of the Euclidean approach, is to investigate its phase structure in the continuum limit, either by numerical simulations or by solving it explicitly.

Before embarking on the physically relevant case of $d$ $=4$, we will in this paper investigate Lorentzian quantum gravity in three dimensions. (Some of the results presented here have been announced recently in $[10,11]$.) Apart from being a new statistical model of three-dimensional fluctuating geometries, there is also some interest from the point of view of quantum gravity proper. Although largely an unphysical theory, 3D quantum gravity is an extensively studied system $[12,13]$. It is often invoked as a model system for the full theory, since its classical equations resemble in many ways those of general relativity. The big difference from $d$ $=4$ is the fact that there are no propagating physical field degrees of freedom. After solving the constraints of the theory, only a finite-dimensional phase space remains. Although one has not yet been able to make full use of this observation in a configuration space path-integral formulation, it suggests that one may still be able to solve 3D gravitational models analytically.

The derivation of the partition function and the explicit construction of 3D Lorentzian simplicial space-times was given in [5]. In order to make this paper self contained, we will summarize the main results below and at the beginning of Sec. III. The Einstein action of a given (smooth) Lorentzian geometry in three dimensions is

$$
S\left[g_{\mu \nu}\right]=\frac{1}{16 \pi G} \int d^{3} x \sqrt{-\operatorname{det} g}(R-2 \Lambda),
$$

where $G$ and $\Lambda$ denote the gravitational and cosmological constants. For the continuous, piecewise linear geometries employed in our simplicial discretization, we use the Regge form of the action [2,14], expressed purely in terms of geometric (coordinate-independent) data, namely, the geodesic edge lengths of the simplicial complexes.

In order to make the state sum well defined, we analytically continue the Regge action associated with each 3D Lorentzian triangulation to Euclidean form by changing the length assignments of all time-like edges from $l_{\text {time }}^{2}=-a^{2}$ to $l_{\text {time }}^{2}=+a^{2}$. The main aim of this paper is to analyze the phase structure of the model defined by the path integral obtained after this "Wick rotation": 


$$
\begin{aligned}
\sum_{\mathcal{T}_{T}\left(S^{1} \times S^{2}\right)} & \frac{1}{C(T)} e^{i S\left(N_{0}, N_{3}, T\right)} \\
l_{\text {time }}^{2} & \rightarrow-l_{\text {time }}^{2} \\
& \mapsto \sum_{\mathcal{T}_{T}\left(S^{1} \times S^{2}\right)} \frac{1}{C(T)} e^{-S_{E}\left(N_{0}, N_{3}, T\right)},
\end{aligned}
$$

where $C(T)$ is the order of the symmetry group of the triangulation $T$. The "measure factor" $1 / C(T)$ appears naturally in the counting of unlabeled triangulations [15]. As usual in the theory of critical phenomena, we do not expect the detailed choice of the measure to affect the continuum limit of the theory, a behavior that has already been corroborated by the 2D models of Euclidean and Lorentzian quantum gravity. In Eq. (3), the Euclidean three-dimensional Regge action $S_{E}$ is expressed in terms of the total numbers of vertices and tetrahedra, $N_{0}$ and $N_{3}$, according to

$$
S_{E}\left(N_{0}, N_{3}, T\right)=-k_{0} N_{0}+k_{3} N_{3},
$$

with the associated dimensionless bare coupling constants

$$
k_{0}=\frac{a}{4 G}, \quad k_{3}=\frac{a^{3} \Lambda}{48 \sqrt{2} \pi G}+\frac{a}{4 G}(3 \kappa-1)
$$

(see Appendix A for a derivation). This form of the action is familiar from former work in Euclidean dynamical triangulations [16]. The parameter $k_{0}$ is proportional to the bare inverse gravitational coupling constant, while $k_{3}$ is a combination of the bare gravitational and cosmological constants [often referred to-somewhat imprecisely-simply as the (bare) cosmological constant]. The geodesic "lattice spacing" (edge length) is given by $a>0$ and $\kappa \pi=\arccos (1 / 3)$ is the dihedral angle of an equilateral tetrahedron.

To keep things simple, we are assuming that the spatial slices have the topology of two-dimensional spheres. In addition, for the convenience of the numerical simulations, we are using periodic boundary conditions in the (Euclidean) time direction, unless specified otherwise. The sum in Eq. (3) is taken over the set of all causal triangulations $\mathcal{T}_{T}\left(S^{1} \times S^{2}\right)$ compatible with this topology, and constructed according to the rules described in Sec. II below. The integer parameter $T$ denotes the total extent in (discrete) proper time, i.e., the number of spatial slices of constant $t \in[0, T]{ }^{1}$

In this paper we explore the phase diagram of threedimensional discrete Lorentzian gravity. We are particularly interested in identifying those regions of coupling-constant space where a continuum limit may exist. This is done with the help of Monte Carlo simulations of the statistical ensemble defined by Eq. (3), combined with qualitative analytical arguments.

The rest of this paper is organized as follows. Section II contains some general considerations on the behavior of discrete quantum gravity models under renormalization. In Sec. III we describe the implementation of the Monte Carlo algo-

\footnotetext{
${ }^{1}$ We have slightly changed our notation with respect to [5], where the total proper time was called $t$.
}

rithm on the ensemble $\mathcal{T}_{T}\left(S^{1} \times S^{2}\right)$ of causal 3D geometries, as well as a characterization of the triangulations and the Monte Carlo moves in terms of dual graphs. Our numerical results are presented in Sec. IV. We characterize the different phases by measuring various geometric observables, and give a detailed description of the geometric properties of the physically interesting "extended" phase. Finally, Sec. V contains a summary and discussion of our results. There are three appendices. In Appendix A, various identities and derivations for 3D simplicial geometries are collected, Appendix B contains more details on dual graphs, and Appendix C some technical specifications of the Monte Carlo simulation.

\section{RENORMALIZATION IN DISCRETE QUANTUM GRAVITY}

As we know from the study of Euclidean simplicial quantum gravity [16], there is a well-defined strategy to search for possible continuum limits for the type of discretized gravity model we are considering. For each value $k_{0}$ of the bare inverse gravitational coupling there is a critical value $k_{3}^{c}\left(k_{0}\right)$ of the bare cosmological constant such that the model is well defined for $k_{3}>k_{3}^{c}\left(k_{0}\right)$ and diverges for $k_{3}<k_{3}^{c}\left(k_{0}\right)$. One can hope to obtain a continuum limit for $k_{3} \rightarrow k_{3}^{c}\left(k_{0}\right)$ because in this limit the expectation value $\left\langle N_{3}^{n}\right\rangle$ may diverge for suitable powers $n$.

This program has been carried out successfully in both Euclidean and Lorentzian quantum gravity in $d=2$ (see [17] for a recent review). It offers a nonperturbative fieldtheoretical definition of $2 \mathrm{D}$ quantum gravity where the bare cosmological constant $k_{2}$ is additively renormalized according to

$$
k_{2}=k_{2}^{c}+\Lambda a^{2}
$$

with $\Lambda$ denoting the $2 \mathrm{D}$ continuum cosmological constant, and where the critical $k_{2}^{c}$ comes entirely from the entropy of the two-dimensional triangulations. We expect an analogous additive renormalization of the bare cosmological coupling constant $k_{3}$ in $3 \mathrm{D}$ quantum gravity, but in this case $k_{3}^{c}$ depends not only on the entropy of the three-dimensional triangulations but also on the gravitational coupling $k_{0}$, since the Einstein action gives a nontrivial weight to each triangulation (contrary to two dimensions, where the curvature term is topological).

Taking the infinite-volume limit of a regularized quantum field theory does not necessarily lead to a continuum quantum field theory. For example, the Ising model on a infinite two-dimensional lattice will only represent a $c=1 / 2$ conformal field theory if at the same time the temperature (which plays the role of a coupling constant in the theory) is fine tuned to the critical temperature of the Ising model. Only when approaching the critical temperature will the longrange spin fluctuations become important and allow us to forget about the details of the lattice regularization, thereby making contact with continuum physics.

By contrast, 2D quantum gravity is an example where the infinite-volume limit of the regularized theory automatically leads to the continuum theory. This was to be expected as the 
cosmological coupling is the only coupling constant of the theory, and at the same time conjugate to the space-time volume.

It is not immediately clear what to expect in threedimensional quantum gravity. The classical theory (after gauge fixing) has no propagating field degrees of freedom, but is described by a finite number of (Teichmüller) parameters, whose number depends on the topology of the spatial slices. Since in the theory of critical phenomena a divergent correlation length and the associated fine tuning of a coupling constant are usually associated with a field degree of freedom, it is tempting to conjecture that the situation will be as in two dimensions, namely, that the infinite-volume limit of 3D Lorentzian quantum gravity (obtained by fine tuning the cosmological constant) coincides with the continuum limit, without the need for further fine tuning.

In three dimensions, we must in addition understand which role the gravitational coupling constant plays in our formulation. In the exponentiated action, it multiplies the curvature term $\int d^{3} x \sqrt{\operatorname{det} g(x)} R(x)$ of the classical Einstein action, from which the classical dynamics is derived. Moreover, it is exactly this term that gives rise to the nonrenormalizability of three-dimensional quantum gravity, when one considers perturbation theory around a classical solution. This means that it does not make much sense to expand around a given flat background in a conventional way. Although the underlying quantum theory may not have any divergences (since there may not be any propagating field degrees of freedom), we are likely to end up with a infinite set of divergent counterterms, ${ }^{2}$ unless there is some as yet undiscovered cancellation mechanism.

There are well-known ways to circumvent this deadlock in the case of three-dimensional gravity, for example, by quantizing in the reduced, finite-dimensional phase space, either in a geometric formulation using $g_{\mu \nu}$ or using gaugetheoretic (Chern-Simons) variables [12,13]. (However, it should be remembered that even classically, the relation between these two "time-full" and "time-less" formulations is only partially understood [18].) How this is reflected in a path-integral quantization in terms of geometries is much less clear (we mean here a configuration space path integral, rather than a phase space path integral). As far as we know, there is not even a generally accepted answer to whether or not the gravitational coupling constant should be renormalized.

If our discretized nonperturbative model possesses a continuum limit, it should provide an answer to this question. For example, the presence of a nontrivial second-order phase

\footnotetext{
${ }^{2}$ One could try to view such a theory as an effective low-energy theory with limited predictive power, much in the same way as the nonlinear sigma model is used as an effective field theory for pion physics, describing some aspects of low-energy QCD. However, this is not what we are after in a nonperturbative definition of $3 \mathrm{D}$ quantum gravity. We want a theory whose predictions in the continuum limit are all independent of the cutoff of the regularized theory, although they may in principle depend on a nonperturbatively induced mass scale.
}

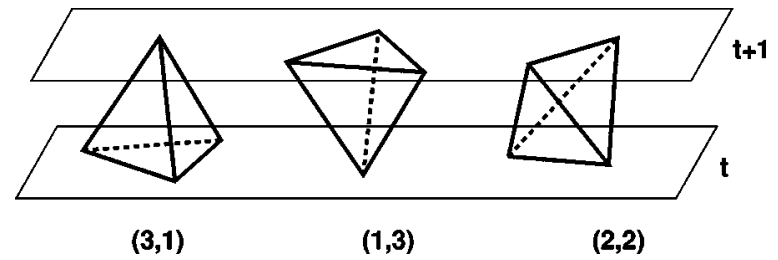

FIG. 1. The three types of tetrahedral building blocks used in discrete 3D Lorentzian quantum gravity, and their location with respect to the spatial slices of constant integer $t$.

transition at a specific value of $k_{0}$ would strongly suggest taking the continuum limit by fine tuning $k_{0}$ to this point, defining in the process the renormalization of the gravitational constant. The issue of nonrenormalizability could be circumvented if the fixed point was nontrivial, not allowing for a simple expansion in geometry. To some extent this is realized in $2+\varepsilon$-dimensional quantum gravity, which possesses a nontrivial fixed point [19-21]. Of course the challenge in such a scenario would be to understand how its excitations are related to the degrees of freedom, or rather the lack of degrees of freedom, of the classical 3D gravity theory. Conversely, if no second- or higher-order transition is present and one can still define a continuum limit, it is likely that no renormalization of the gravitational constant is necessary.

In order to find answers to these questions, we will in this paper analyze data coming from numerical studies of threedimensional Lorentzian gravity. Attempts to solve the model analytically are underway and will be reported elsewhere [22]. As will be described in the following, our investigation provides evidence that (i) a continuum limit exists, (ii) there is a well-defined ground state which dominates the functional integral and thus represents a "background geometry," and (iii) the bare gravitational coupling constant sets a length scale for the geometry, but is not renormalized.

\section{NUMERICAL IMPLEMENTATION OF THE MODEL}

Which are the three-dimensional Lorentzian geometries contributing to the state sum of Eq. (1)? Starting from a sequence of two-dimensional equilateral triangulations, a three-dimensional Lorentzian triangulation is obtained by filling the spaces between pairs of such spatial slices by three types of tetrahedral building blocks, in such a way as to form a simplicial manifold. They are: (i) the so-called $(3,1)$ tetrahedra with a triangle in the spatial $t$ plane and a vertex in the spatial $t+1$ plane; their number in any given sandwich $[t, t$ $+1]$ is denoted by $N_{31}(t)$, and their total number by $N_{31}$; (ii) the $(1,3)$ tetrahedra with a vertex in the $t$ plane and a triangle in the $t+1$ plane; their number in any given sandwich $[t, t+1]$ is denoted by $N_{13}(t)$, and their total number by $N_{13}$; and (iii) the $(2,2)$ tetrahedra with one link in the $t$ plane and another one in the $t+1$ plane; their number in any given sandwich $[t, t+1]$ is denoted by $N_{22}(t)$, and their total number by $N_{22}$ (see Fig. 1).

Several of our numerical measurements involve the twodimensional volume of the spatial slices. In our model there are two natural ways of defining the spatial volume at time $t$. 


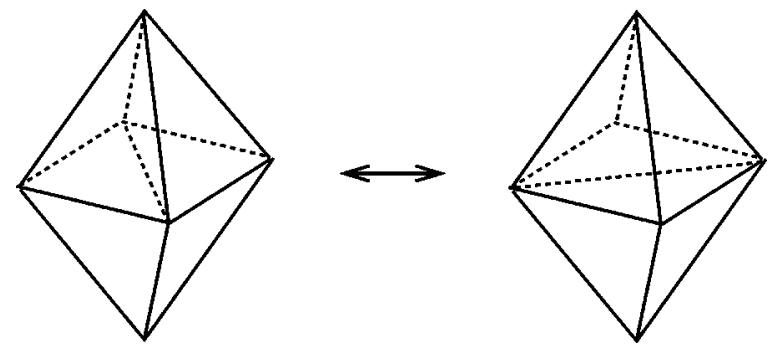

FIG. 2. In a flip move, the four tetrahedra inside a diamond configuration are reassigned by flipping the diagonal of the central square.

One can define it simply as the number of triangles in the spatial slice of constant integer $t$

$$
N_{2}^{(s)}(t) \equiv N_{31}(t) \equiv N_{13}(t-1)
$$

or measure it at half-integer $t$ and define $\mathrm{e}^{3}$

$$
N_{2}^{(s+1 / 2)}(t):=N_{13}(t)+N_{31}(t)+N_{22}(t)
$$

In a phase of extended geometry (such that $N_{13} \sim N_{31} \sim N_{22}$ ), both definitions should lead to equivalent results. For most purposes, we have found it convenient to work with the two volumes $N_{2}^{(s+1 / 2)}(t)$.

We will explore the infinite-volume limit of the ensemble of discrete Lorentzian geometries by performing a Monte Carlo simulation where each suggested local change of triangulation (a "move") is accepted or rejected according to certain probabilities depending on the change in the action and the local geometry.

Our local updating algorithm consists of five basic moves. They change one Lorentzian triangulation into another, while preserving the constant-time slice structure, as well as the total proper time $T$. We are confident that this set of moves is ergodic in the space of all allowed Lorentzian triangulations at fixed $T$, although we do not as yet have complete formal proof. Note that all of the moves described below will be rejected in the updates if they lead to triangulations where pairs of vertices are connected by more than one link or where triplets of vertices belong to more than one triangle, since this violates the simplicial manifold property. Let us now describe each of the moves in turn.

(1) Consider two neighboring triangles in the spatial $t$ plane. Each of them belongs to a tetrahedron above and below that plane. Assume now that both the two $(3,1)$ tetrahedra above and the two $(1,3)$ tetrahedra below share a triangle. Together, the four tetrahedra form a diamond whose tips lie in the $t-1$ and in the $t+1$ plane, and whose intersection with the $t$ plane is a square. The move consists of flipping the link that forms the diagonal of this square to the opposite

\footnotetext{
${ }^{3}$ This definition is the simplest one in that it counts the number of building blocks at $t=1 / 2$. We could put in individual weights reflecting the actual areas of the cross sections of the tetrahedra, but it would not affect our results below.
}

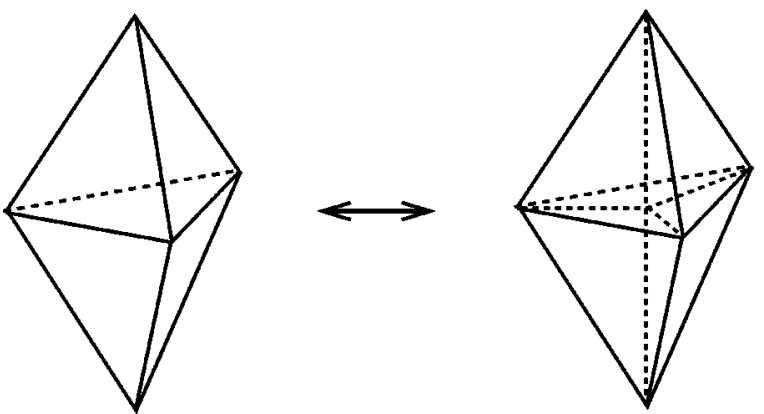

FIG. 3. Insertion or removal of a vertex in the central triangle transforms two into six tetrahedra and vice versa.

diagonal, accompanied by the corresponding reassignment of the tetrahedra constituting the diamond (Fig. 2).

(2) (3) Consider a triangle in the $t$ plane together with its two neighboring tetrahedra, whose two tips $v_{t+1}$ and $v_{t-1}$ lie in the $t+1$ and the $t-1$ planes. We can always insert a vertex $v_{t}$ at the center of the triangle and connect it to the exterior vertices of this configuration by adding five internal links, thus replacing the original two tetrahedra by six (Fig. 3 ). The corresponding inverse move can only be performed if we can identify a vertex $v_{t}$ of order six (i.e., belonging to six tetrahedra), together with two links $\left(v_{t}, v_{t-1}\right)$ and $\left(v_{t}, v_{t+1}\right)$ which are both of order three. In this case one can just remove $v_{t}$ and both links $\left(v_{t}, v_{t \pm 1}\right)$, replacing in an obvious way the six tetrahedra by two.

(4) (5) The fourth move can be performed on any configuration consisting of a pair of a $(2,2)$ and a $(3,1)$ [or a $(1,3)$ ] tetrahedron having a triangle in common. We can remove the triangle (but not its links and vertices) and insert a link dual to it, connecting the two vertices which did not belong to the triangle (see Fig. 4). In this way the original $(3,1)$ and $(2,2)$ tetrahedra are replaced by one $(3,1)$ and two $(2,2)$ tetrahedra, without introducing any changes in the two-dimensional spatial slices. The fifth move is the inverse of the fourth move, replacing a suitable configuration of one $(3,1)$ [or $(1,3)]$ tetrahedron and two adjacent $(2,2)$ tetrahedra by a pair of a $(3,1)[$ or $(1,3)]$ and a $(2,2)$ tetrahedron.

Note that not all of the local moves preserve the three volume. We will use a standard way of dealing with this situation, developed for dynamically triangulated models in dimensions three and four [16]. This method ensures that the volume of the system fluctuates around a prescribed value $N_{3}$, with a well-defined range of fluctuations.

In the implementation of the numerical code it is convenient to work not with the triangulations and their constituents but with the dual graphs, which are given by specific

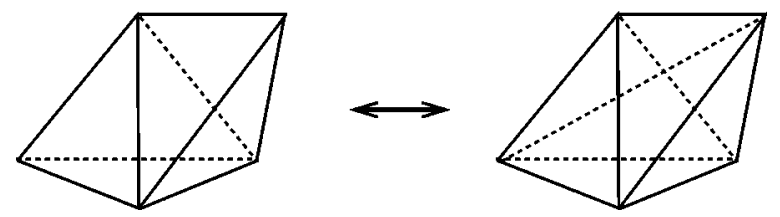

FIG. 4. A neighboring pair of a $(3,1)$ and a $(2,2)$ tetrahedron is replaced by another $(3,1)$ tetrahedron (whose tip lies now in the top right-hand corner) and a $(2,2)$ tetrahedron on either of its flanks. 

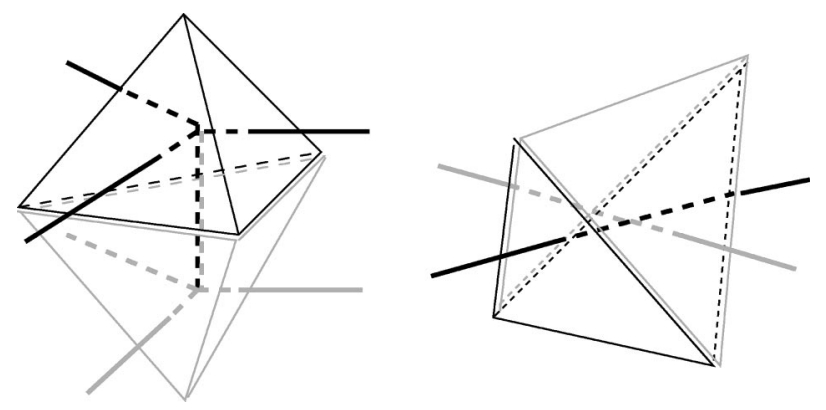

FIG. 5. How the tetrahedral building blocks give rise to dual bicolored graphs (drawn as gray and black horizontal lines) at halfinteger $t$.

classes of $\phi^{4}$ graphs. Like the triangulations, all of the graphs have a foliated structure. This is most naturally associated with half-integer times, because the vertices of the dual graph are located at the centers of the tetrahedra of the original triangulation. To visualize the geometry of the gluings and the moves in this dual language, we adopt a coloring for the dual graphs. A link dual to a triangle of a $(3,1)$ tetrahedron is "black," and one dual to a triangle of a $(1,3)$ tetrahedron is "gray." This results in a unique coloring for all links dual to "time-like" triangles (lying inbetween spatial slices), since it is not possible to directly glue a gray to a black triangle. This can only be done if the triangles are space-like (i.e., if they are both contained in the same slice $t=$ const). The links dual to such triangles are therefore double colored (Fig. 5, left). We can now construct for each "sandwich," i.e., each triangulated space-time slice $[t, t$ +1 ], a bicolored graph (with topology $S^{2}$ ) by projecting all unicolored dual links associated with the sandwich to the plane at $t+1 / 2$.

In this way each $(3,1)$ tetrahedron gives rise to three black links, sharing a trivalent intersection. Each of the links can end at the center of either another $(3,1)$ tetrahedron or a $(2,2)$ tetrahedron, but never at the center of a $(1,3)$ tetrahedron. [An analogous statement holds for the triplet of gray links associated with a $(1,3)$ tetrahedron.] Consequently, each $(2,2)$ tetrahedron in the sandwich corresponds to a four-valent vertex of the dual graph, with alternate colors black-gray-blackgray for the incoming links (Fig. 5, right). The end result is a combined gray-and-black graph in the $t+1 / 2$ plane. Moreover, each such graph occurs in the large- $n$ limit of the perturbative expansion of the two-matrix model defined by the partition function

$$
\begin{aligned}
Z\left(\alpha_{G}, \alpha_{B}, \beta\right)= & \int d \phi_{G} d \phi_{B} \exp n \operatorname{tr}\left[-\frac{1}{2}\left(\phi_{G}^{2}+\phi_{B}^{2}\right)\right. \\
& \left.+\frac{\alpha_{G}}{3} \phi_{G}^{3}+\frac{\alpha_{B}}{3} \phi_{B}^{3}+\frac{\beta}{2} \phi_{G} \phi_{B} \phi_{G} \phi_{B}\right],
\end{aligned}
$$

where, as usual, the quadratic terms give rise to propagators or links, and the cubic and quartic interaction terms correspond exactly to the tri- and four-valent intersections illustrated in Fig. 5. Note that not all graphs generated by Eq. (9)
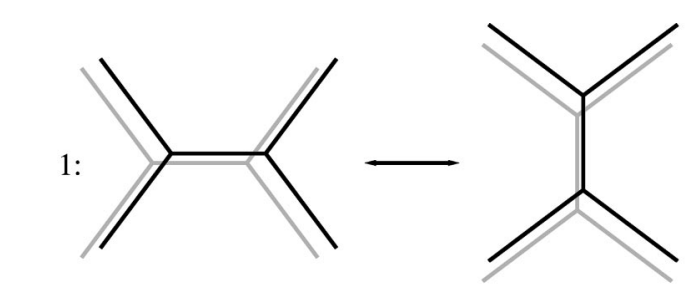

2\&3:
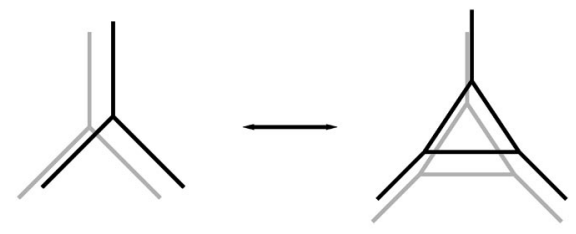

4\&5:
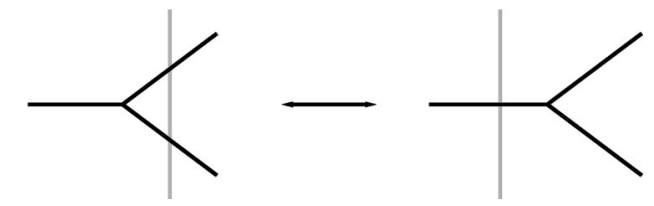

FIG. 6. Graphical illustration of the Monte Carlo moves in terms of dual bicolored graphs. In moves 1 and 2 and 3, dual links from two adjacent sandwiches are affected. Moves 4 and 5 take place within a given $[t, t+1]$ sandwich.

correspond to allowed Lorentzian triangulations, since their duals may violate the 3D simplicial manifold constraints. This matrix model (with some additional assumptions about universality) can be taken as the starting point for an analytical solution of the transfer matrix of simplicial 3D quantum gravity [22].

The time evolution in the dual picture can be thought of as follows. A bicolored graph at time $t+1 / 2$ consists of two components: a black $\phi^{3}$ graph dual to the triangulation at time $t$, and a gray $\phi^{3}$ graph dual to the triangulation at $t$ +1 . The way in which the two original spatial triangulations are glued together is encoded in the intersection pattern of the "superposition" of the two graphs at time $t+1 / 2$. If we view the black and gray trivalent graphs as representing in and out states, their transition amplitude is a function of the number of topologically inequivalent ways of superposing the two graphs (subject to some "dual" manifold constraints—see Appendix B for details).

The five Monte Carlo moves described earlier can also be rephrased in the language of intersecting colored $\phi^{3}$ graphs, as illustrated in Fig. 6. The diagrams appearing in Fig. 6 contain all dual links affected by a given move. Since the moves 1, 2, and 3 are symmetric with respect to the plane $t=$ const, a change in one of the trivalent graphs is always accompanied by an equivalent change of its mirror image of the opposite color. Moves 4 and 5 assume a particularly simple form: one link of a given color is "dragged across" a vertex of the opposite color. [Note that in the graphical representation of this particular move, the cubic vertices represent $(3,1)$ or $(1,3)$ tetrahedra and the black-gray crossings $(2,2)$ tetrahedra.] Details about the numerical implementation in terms of this dual picture (including lattice sizes, update efficiency, number of sweeps, etc.) can be found in Appendices $\mathrm{B}$ and $\mathrm{C}$. 


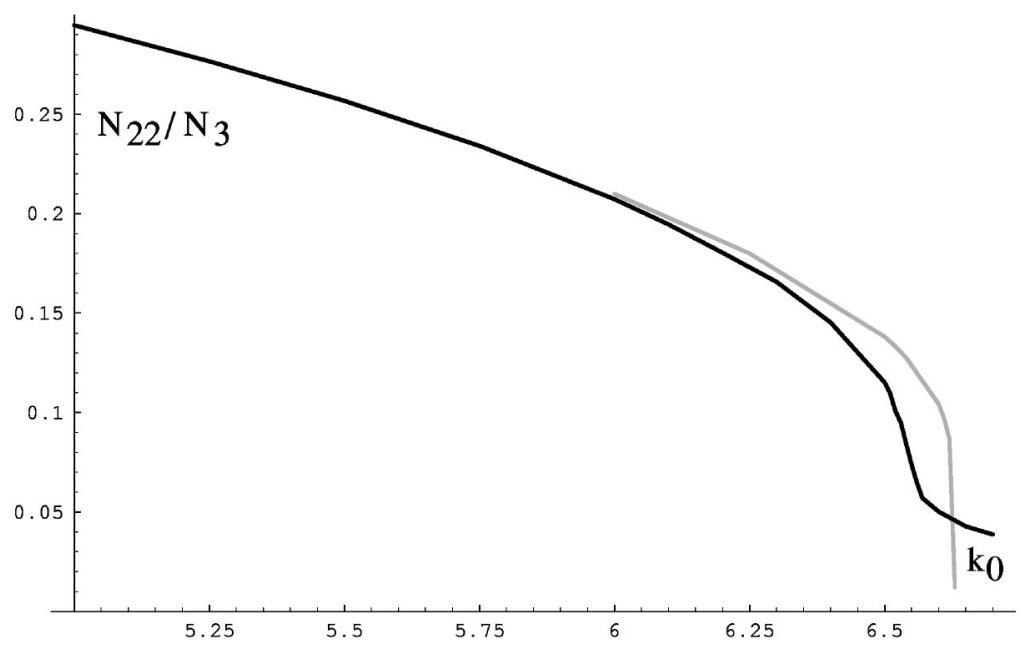

FIG. 7. The order parameter $\tau=N_{22} / N_{3}$ for configurations with $T=64$, and $N_{3}=16000$ (long dark curve) and 64000 (short light curve), plotted as a function of $k_{0}$. The curve is a linear interpolation between data points. (Error bars smaller than width of curve.)

\section{NUMERICAL RESULTS}

Having presented our numerical setup, we are now in a position to extract a number of physical properties of the Lorentzian model. We will first investigate the phase diagram of the regularized theory, and try to understand which of the continuum-limit scenarios outlined in Sec. II is realized. We will then analyze the geometry characterizing the different phases. Since we have a distinguished (and coordinateinvariant) notion of proper time $t$, we can extract invariant information of the system by studying correlation functions in $t$. This will be done by measuring distributions of spatial slice volumes $N_{2}^{(s+1 / 2)}$ as a function of the total proper time $T$ and correlators $\left\langle N_{2}^{(s+1 / 2)}\left(t_{1}\right) N_{2}^{(s+1 / 2)}\left(t_{2}\right)\right\rangle$ between spatial volumes, as well as the intrinsic Hausdorff dimension $d_{H}^{\text {sp }}$ of a typical spatial slice.

\section{A. The phase diagram}

In order to explore the phase diagram of the regularized Lorentzian model we must find an order parameter, and explore how it changes with the coupling constant, in this case $k_{0}$. We have found that the ratio between the total number $N_{22}$ of $(2,2)$ tetrahedra and the total space-time volume $N_{3}$

$$
\tau=\frac{N_{22}}{N_{3}} \equiv \frac{N_{22}}{N_{22}+N_{31}+N_{13}}
$$

serves as an efficient order parameter. We shall not be concerned with a continuum interpretation of this parameter (which is not obvious) since we will go on to show that no continuum physics is associated with the transition we observe as a function of $\tau$. In Figs. 7 and 8 we show the ratio $\tau$ as a function of $k_{0}$ for two different types of space-time configurations. In Fig. 7 all geometries have 64 spatial slices $(T=64)$, with total space-time volumes $N_{3}=16000$ and $N_{3}=64000$. One observes a rapid drop to zero of $\tau\left(k_{0}\right)$ around $k_{0} \approx 6.64$. Increasing $N_{3}$, the drop becomes a jump, characteristic for a first-order phase transition. A detailed study of the neighborhood of $k_{0}=6.64$ reveals a (weak) hysteresis as one performs a cycle, moving above and below the critical value $k_{0}^{c}$, again as expected in a first-order transition.
The location of the phase transition depends weakly on the total length $T$ in time direction, and the jump in $\tau$ becomes more pronounced for larger volumes. Figure 8 is an illustration of the extreme case $T=1$, where we have chosen free instead of periodic boundary conditions (the triangulations at the initial time $t=1$ and the final time $t=2$ are allowed to fluctuate freely), so that the topology of space-time is changed to $S^{2} \times[0,1]$. $^{4}$

As can be read off from Fig. 7 , for $k_{0}>k_{0}^{c} \approx 6.64$ only a minimal number of $(2,2)$ tetrahedra is present. This can be understood by rewriting the action Eq. (4) to make the dependence on $N_{22}$ explicit. In Appendix A we derive

$$
S_{E}=\frac{k_{0}}{4} N_{22}+\left(k_{3}-\frac{k_{0}}{4}\right) N_{3}-2 k_{0} T,
$$

which shows that for fixed $N_{3}$ and $T$ (and positive gravitational coupling $k_{0}$ ) a minimal $N_{22}$ corresponds to a minimum of the Euclidean action.

The entropy of configurations with $N_{22}$ different from its minimal value will in general ensure that the ratio $\tau$ is different from zero, even when $N_{3} \rightarrow \infty$. However, since the number of such configurations for fixed $N_{3}$ grows at most exponentially with $N_{3}$, this leaves the possibility that for sufficiently large $k_{0}$ the term $e^{-k_{0} N_{22} / 4}$ will dominate over the entropy contribution and trigger a phase transition to a phase with only a minimal number of $(2,2)$ tetrahedra, such that $\tau$ $=0$ in the continuum limit.

The physics of this phase can be readily understood. In terms of the matrix model, a situation with no $(2,2)$ tetrahedra corresponds to choosing the coupling constant $\beta=0$ in Eq. (9), thus reducing the model to a product of two independent $\phi^{3}$ matrix models. Since a $\phi^{3}$ matrix model at its critical point describes two-dimensional Euclidean quantum gravity, the matrix model analogy strongly suggests that the Lorentzian 3D model for $k_{0}>k_{0}^{c}$ can be viewed as a product of uncoupled 2D gravity models. This conclusion seems to

\footnotetext{
${ }^{4}$ In this situation the critical point is changed from $k_{0}^{c}=6.64$ to $k_{0}^{c}=6.42$.
} 


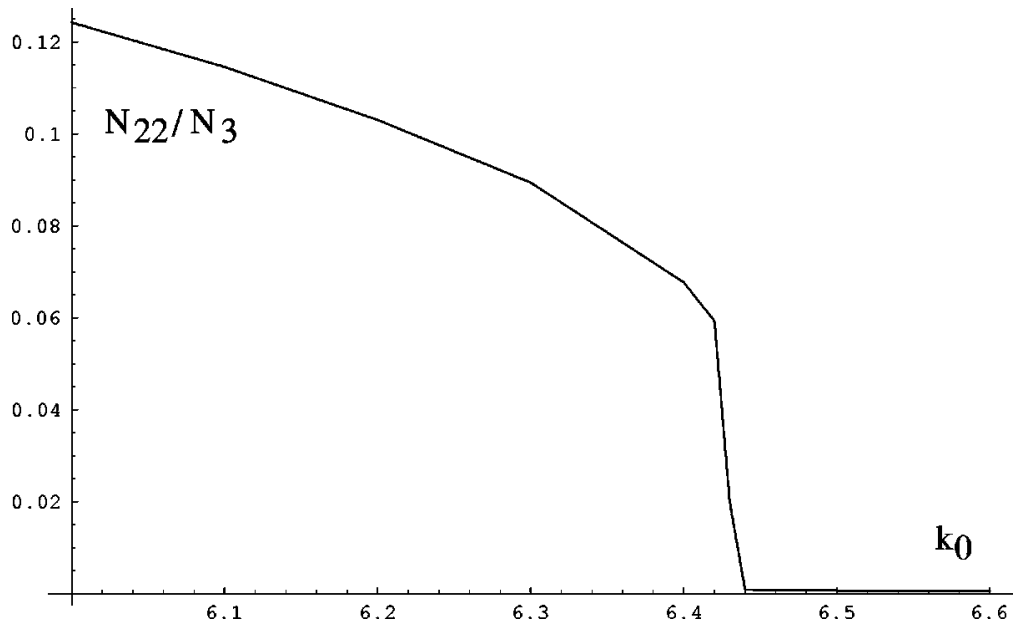

FIG. 8. The order parameter $\tau=N_{22} / N_{3}$ for configurations with $T=1$ and $N_{3}=16000$, but with free boundary conditions, plotted as a function of $k_{0}$. The curve is a linear interpolation between data points. (Error bars smaller than the width of the curve.)

be corroborated by our numerical results. Figure 9 is a typical "snapshot" of a space-time geometry, taken during the computer simulations. The spatial volume $N_{2}^{(s+1 / 2)}(t)$ is shown as a function of the time $t$. Apparently it can change from essentially zero to a "macroscopic" size in a single time step, which implies that there cannot be any correlations between slices separated by a few time steps. A different measurement of the correlation between successive spatial volumes is depicted in Fig. 10. We have again chosen $T$ $=1$ and free boundary conditions, in order to have spatial slices of a reasonably large size, but a qualitatively similar behavior is expected for $T>1$ too. We show the distribution of the (normalized) difference

$$
\begin{aligned}
\widetilde{\tau}(1 \rightarrow 2) & =\frac{\left|N_{2}^{(s)}(t=2)-N_{2}^{(s)}(t=1)\right|}{N_{3}} \\
& \equiv \frac{\left|N_{2}^{(s)}(2)-N_{2}^{(s)}(1)\right|}{N_{31}(1)+N_{13}(1)+N_{22}(1)}
\end{aligned}
$$

of the spatial volumes of the initial and final slice. For $k_{0}$ less than the critical $k_{0}^{c} \approx 6.42, \widetilde{\tau}$ is peaked around zero. The peak becomes flatter as $k_{0}$ approaches its critical value and immediately beyond $k_{0}^{c}$, the distribution approximates a $\delta$ function around $\widetilde{\tau}=1$.

This last result can be understood as follows. We know from the simulations that the number of $(2,2)$ tetrahedra drops to a minimum beyond the critical point $k_{0}^{c}$. To first approximation, these tetrahedra therefore do not contribute to the entropy in that region. Moreover, since a minimal set of $(2,2)$ tetrahedra can basically interpolate between any pair of "incoming" $(3,1)$ and "outgoing" $(1,3)$ configurations, the combinatorics is governed by the separate countings of those configurations, subject only to an overall volume constraint $N_{31}+N_{13}=N_{3}-N_{22} \approx N_{3}=$ const. Individually, the configurations at $t=1$ and $t=2$ are simply 2D Euclidean triangulations, whose number for a given spatial volume $N_{2}^{(s)}$ is known to be proportional to $e^{c N_{2}^{(s)}}\left(N_{2}^{(s)}\right)^{-5 / 2}$. From $\widetilde{\tau}(1$ $\rightarrow 2)=\left|1-2 N_{13} / N_{3}\right|$, and taking into account that the minimal interpolating $N_{22}$ configuration can be inserted anywhere in the incoming and outgoing configurations, one finds

$$
\begin{aligned}
\#\left(N_{31} \rightarrow N_{13}\right) & \sim e^{c N_{31}} N_{31}^{-(3 / 2)} e^{c\left(N_{3}-N_{31}\right)}\left(N_{3}-N_{31}\right)^{-(3 / 2)} \\
& \sim\left(1-\widetilde{\tau}^{2}\right)^{-(3 / 2)}
\end{aligned}
$$

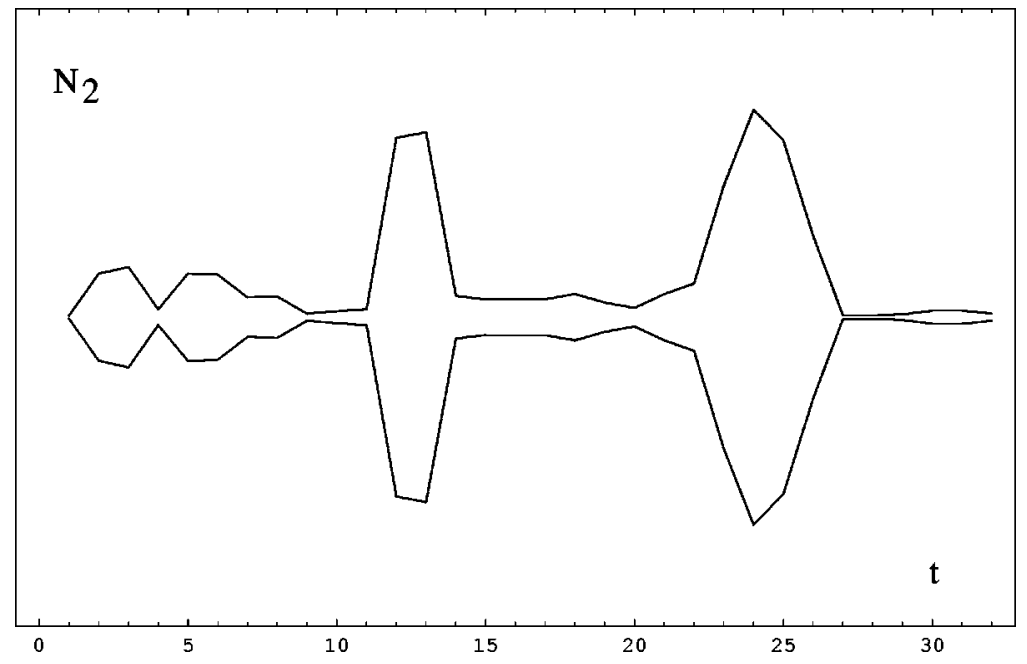

FIG. 9. Monte Carlo snapshot of the distribution of spatial volumes $N_{2}^{(s+1 / 2)}(t)$, for $T=32$, $N_{3}=16000$, and $k_{0}=6.7$ (that is, above the critical value $\left.k_{0}^{c}=6.64\right)$. The volumes are plotted symmetrically about a central horizontal $t$ axis. 


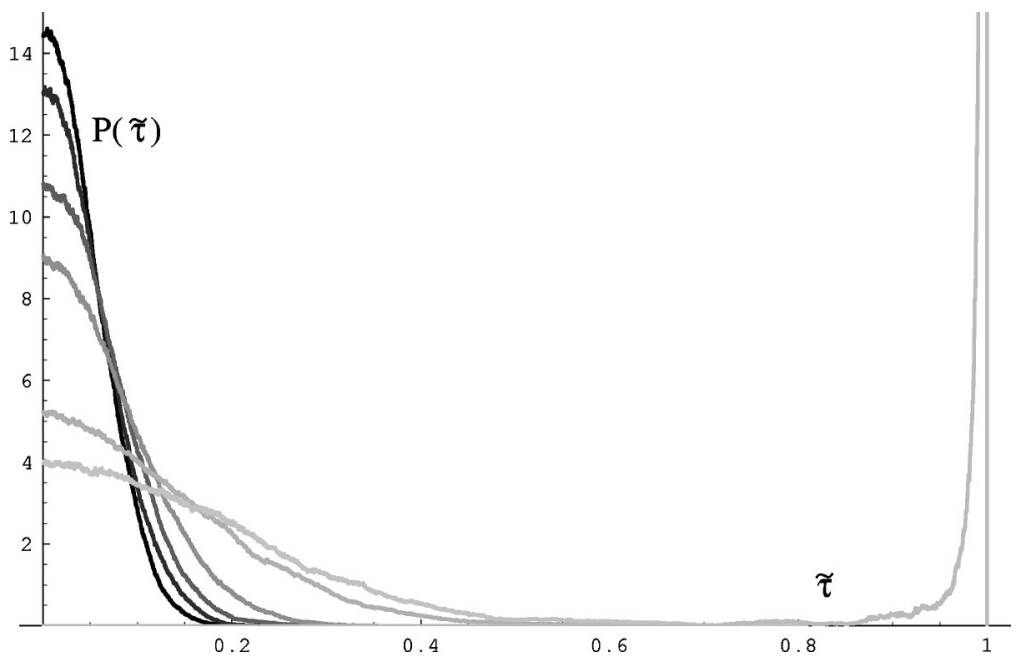

FIG. 10. The probability distribution $P(\widetilde{\tau})$ of $\tilde{\tau}=\left|N_{2}^{(s)}(2)-N_{2}^{(2)}(1)\right| / N_{3}$ for $k_{0}=6.0,6.1,6.2$, $6.3,6.4$, and 6.42 (highest to lowest peak around $\tilde{\tau}=0$; the value $k_{0}=6.42$ corresponds to the phase transition point) and 6.44 (distribution peaked around 1); for total volume $N_{3}=8000$ and free boundary conditions.

for the combined entropy at fixed volume $N_{3}$. In agreement with Fig. 10, it shows that the most likely configurations are those where the entire $3 \mathrm{D}$ volume is concentrated at one of the slices, that is, either $N_{31} \approx 0, N_{13} \approx N_{3}$ or vice versa.

The situation is very different in the phase with $k_{0}<k_{0}^{c}$. Our measurements of $\widetilde{\tau}$ at $T=1$ are an indication that also in general in this phase the volumes of successive spatial slices will be strongly coupled, i.e., their volume difference will be small. Since this behavior is not favored by the action, the prevalence of such configurations must have to do with the presence of the $(2,2)$ tetrahedra and their associated entropy (i.e., the number of ways they can combine with each other and with other tetrahedra to form interpolating "sandwiches"). This observation turns out to be of great importance, since it seems to lie at the heart of the emergence of extended geometries in this phase, which will be described in more detail in the next section. In broad terms, the phase is characterized by $0<\tau<1$. In principle there may be another phase transition at some smaller (possibly negative) value $\tilde{k}_{0}$, such that $\tau=1$ for $k_{0}<\widetilde{k}_{0}$ [with no $(3,1)$ or $(1,3)$ tetrahedra surviving in the continuum limit]. Indeed, for fixed $N_{3}$ and negative $k_{0}$ the action Eq. (11) has a minimum for $\tau$ $\approx 1$. (Configurations with $\tau \rightarrow 1$ for $N_{3} \rightarrow \infty$ can actually be realized.)

Whether or not the system will undergo a phase transition for sufficiently small $k_{0}$ will depend on the balance between action and entropy, which cannot be determined by simple qualitative considerations. We have not investigated this region of the coupling constant space further, given the limited importance of negative gravitational coupling constants from a quantum gravity viewpoint, and the fact that our computer algorithm is not efficient at small $k_{0}$.

In summary, we have arrived at the following tentative description of the phase diagram of 3D Lorentzian quantum gravity: the bare inverse gravitational coupling constant has two critical values, $\widetilde{k}_{0}^{c}$ and $k_{0}^{c}$ (possibly with $\widetilde{k}_{0}^{c}=-\infty$ ). For $k_{0}>k_{0}^{c}$ the model describes the fluctuations of an uncorrelated set of two-dimensional spatial geometries and has nothing to do with a three-dimensional theory of gravity. Also for $k_{0}<\widetilde{k}_{0}^{c}$ the space-time geometry degenerates, since the spatial slices at integer $t$ completely disappear from the theory.
These two "extreme" regions of the phase diagram can be regarded as artifacts of our particular way of setting up the discretized theory. They may be seen as remnants of the phases of degenerate geometries observed previously in 3D Euclidean quantum gravity [16]. However, unlike the Euclidean theory, Lorentzian gravity possesses a large region $\widetilde{k}_{0}^{c}$ $<k_{0}<k_{0}^{c}$ of coupling constant space where the quantum geometry is extended and well behaved, and whose description we shall turn to next.

\section{B. The phase of extended geometry}

Let us now analyze the structure of the phase of intermediate gravitational coupling, $\widetilde{k}_{0}^{c}<k_{0}<k_{0}^{c}$, where all types of tetrahedral building blocks contribute nontrivially. Quite remarkably, and unlike in the phase where $k_{0}>k_{0}^{c}$ we observe here the emergence of well-defined three-dimensional configurations. Figure 11 shows a snapshot of a typical geometry at $k_{0}=5.0$, consisting of 16000 tetrahedra, for $T=32$. [As in the previous Fig. 9, we plot-symmetrically around an arbitrary axis - the spatial volume $N_{2}^{(s+1 / 2)}(t)$ as a function of $t$.] Following the computer-time history of this extended object, it is clear that although it does indeed fluctuate, the fluctuations take place around a three-dimensional object of welldefined linear extension. ${ }^{5}$ The emergence of a ground state of extended quantum geometry is a highly nontrivial property of the Lorentzian model, since we have at no stage put in a preferred background geometry by hand. No structures of this kind have ever been observed in dynamically triangulated models of Euclidean quantum gravity. It underscores the fact that the Lorentzian models are genuinely different and affirms our conjecture [5] that in $d \geqslant 3$ they are less pathological than their Euclidean counterparts.

For a fixed $k_{0}$ and $N_{3}$, an overall "spherical" shape as shown in Fig. 11 is found only for sufficiently large $T$. At

\footnotetext{
${ }^{5} \mathrm{~A}$ trivial mode of fluctuations are the translations in timedirection. Due to the periodicity of the boundary condition the "center of volume" of the extended configuration performs a random walk in the $t$ direction.
} 


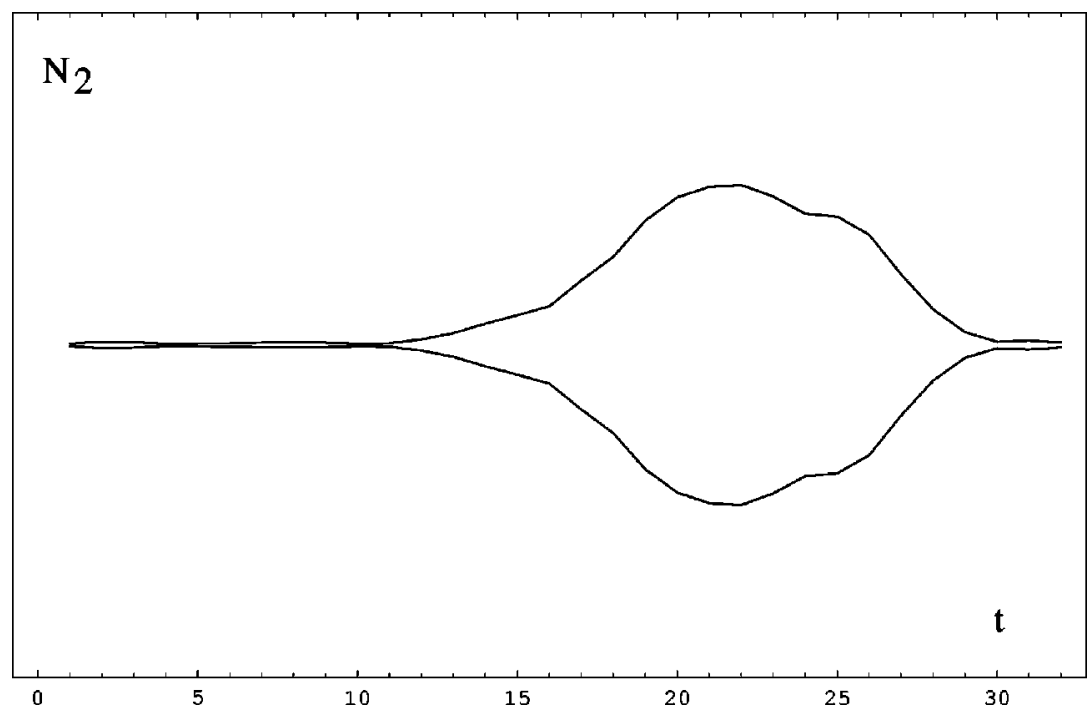

FIG. 11. Monte Carlo snapshot of the distribution $N_{2}^{(s+1 / 2)}(t)$ of spatial volumes, for $T=32, N_{3}$ $=16000$, and $k_{0}=5.0$ (that is, below the critical value $\left.k_{0}^{c}=6.64\right)$. The volumes are plotted symmetrically about a central horizontal $t$ axis.

small $T$, one observes a uniform distribution of spatial volumes $N_{2}^{(s+1 / 2)}(t)$ as a function of $t$. As $T$ increases, the bulk of the space-time volume "condenses" into a region with a well-defined extent $T_{u}<T$ in time-direction (which we will call the universe), leaving over a thin stalk of minimal spatial radius everywhere else along the $t$ axis. We will from now on choose $T$ sufficiently large, so that $T>T_{u}$ for all volumes under consideration. We are interested in the "cosmological" properties of this extended universe, i.e., its geometric properties at large scales. Our data for the scaling of the time extent $T_{u}$ of the universe as a function of the total volume are consistent with

$$
T_{u} \sim N_{3}^{1 / 3}
$$

(We discuss below how a quantitative measure of $T_{u}$ is obtained.) Similarly, by an independent measurement of the volumes $N_{2}^{(s+1 / 2)}(t)$ of spatial slices that lie within the universe, we have found a scaling behavior consistent with

$$
\left\langle N_{2}^{(s+1 / 2)}(t)\right\rangle \sim N_{3}^{2 / 3} .
$$

Relations (14) and (15) support an interpretation of the universe as a genuinely three-dimensional object. There is of course no a priori reason that a ground state in a nonperturbative theory of quantum gravity (if it exists) should bear any resemblance to a (semi-)classical geometry. Let us explain briefly how such geometries (and, more specifically, classical solutions) might still make an appearance in this context.

For the simplest compact space-time topology, the solution to the classical Einstein equations with Euclidean signature and a positive cosmological constant $\Lambda$ is the round three-sphere (of constant positive scalar curvature) with radius $R_{S^{3}} \sim \Lambda^{-1 / 2}$. Solving the equations with the constraint of fixed space-time volume $V$ is equivalent to introducing an effective cosmological constant $\Lambda_{\text {eff }} \sim V^{-2 / 3}$. We are not aware of a classical solution with positive $\Lambda$ and topology $S^{1} \times S^{2}$ (which is the topology used in our simulations). However, for our purposes we can "adapt" the $S^{3}$ solution to this topology by cutting away two small open balls at two opposite points of an $S^{3}$ configuration with radius $R_{S^{3}}$ $\sim \Lambda_{\text {eff }}^{-1 / 2} \sim V^{1 / 3}$ and attaching a thin cylinder $I \times S^{2}$ (with spatial radius at the cutoff scale) to the holes. This will produce a geometry of the kind shown in Fig. 11. Although it is not strictly speaking a solution to Einstein's equations, it is "almost as good" from the point of view of the path integral, since-independent of its metric properties - the contribution of the stalk to the action is negligible (because it does not grow proportionally to the three volume).

Suppose for the moment that the round $S^{3}$ solution corresponded to a (local) minimum of the action. Then the singular "solution" of topology $S^{2} \times S^{1}$ constructed above would also represent a (local) minimum of the action, and would therefore be as relevant as the $S^{3}$ solution in the quantum theory. Unfortunately, the argument is not quite as simple, because the classical continuum Einstein action is unbounded from below, due to the presence of a kinetic term of the "wrong" sign, coming from the conformal mode of the metric. However, since the conformal mode is not a propagating degree of freedom in either classical general relativity or in canonical quantizations, it should not cause any problems in a correct, nonperturbative path-integral quantization of gravity, not even in the Euclidean sector. ${ }^{6}$ In such a quantum theory the effective action should be bounded from below and semiclassical saddle-point considerations of the kind made above may again be appropriate.

We have measured the correlation function

$$
C(\Delta)=\frac{1}{T^{2}} \sum_{t=1}^{T}\left\langle N_{2}^{(s+1 / 2)}(t) N_{2}^{(s+1 / 2)}(t+\Delta)\right\rangle
$$

as a function of the displacement $\Delta$ to determine the scaling of $T_{u}$ with the space-time volume $N_{3}$. This correlator has the advantage of being translation invariant in $t$ and allows for a precise measurement by averaging over many independent

\footnotetext{
${ }^{6}$ The continuum path integral in the proper-time gauge is discussed in [23].
} 


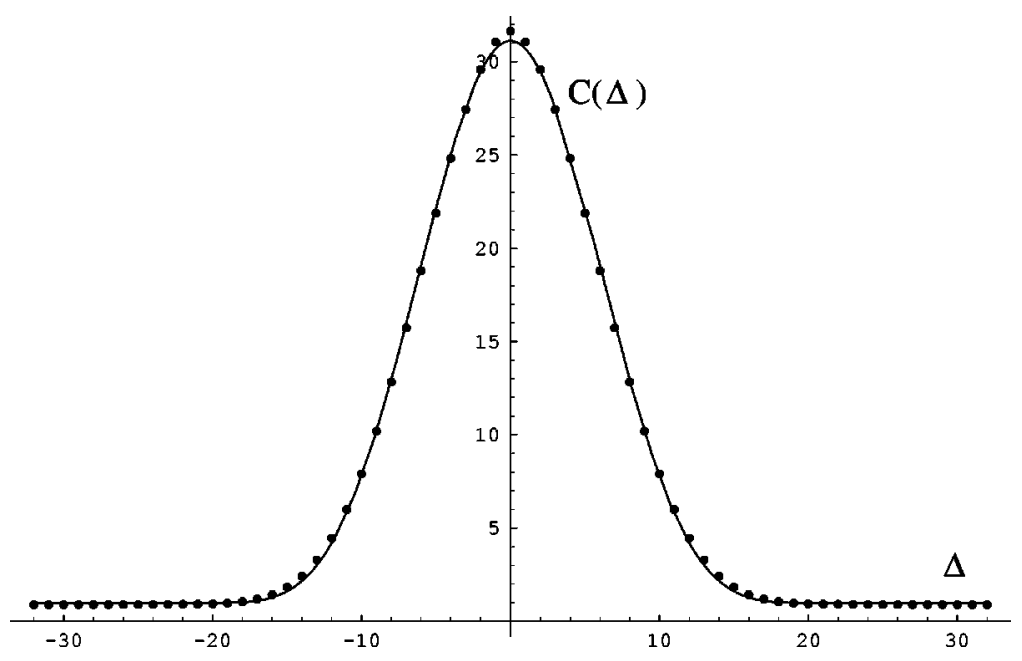

FIG. 12. The correlator $C(\Delta)$ with $T=64$ and $N_{3}=32000$. Dots are the measured values (error bars less than dots), and the curve is fitted from the sphere solution described in the text.

configurations. From the typical shape of the space-time configurations we expect $C(\Delta)$ to be on the order of the spatial cutoff if $\Delta>2 T_{u}$. Figure 12 illustrates the result of our measurements of $C(\Delta)$, with the dots representing the measured values. The theoretical curve to which we are fitting corresponds to the "fake sphere" described above, with the radius of the $S^{3}$ and the spatial radius of the thin cylinder attached to it as free parameters. For this "spherical" geometry we then perform the integral (the sum) in Eq. (16), without the average $\langle\cdot\rangle$. As is evident from Fig. 12, the volume distribution associated with this fixed geometry gives a rather good fit to our data. This provides some evidence that we can ignore the quantum average implied by $\langle\cdot\rangle$, and that our universes behave semiclassically, at least as far as their macroscopic geometric properties are concerned. We should mention that our " $S^{3}$ solution" is not singled out uniquely, since the choice of a Gaussian shape in the $t$ direction gives a fit of comparable quality.

For various space-time volumes $N_{3}$ (typically 8, 16, 32, and $64 k$ ) we have determined the radius $R_{S^{3}}$ of $S^{3}$ from the fits to the measured $C(\Delta)$. From this, we have finally found $\alpha=0.34 \pm 0.02$ as the best exponent in the scaling relation

$$
R_{S^{3}}\left(N_{3}\right)=N_{3}^{\alpha}
$$

The same value is obtained using other ways to extract $T_{u}$, lending additional support to the three-dimensional nature of our universes.

We will now take a closer look at the geometry of the two-dimensional spatial slices. If they could be described as typical triangulated surfaces in 2D Euclidean quantum gravity, they would not behave like smooth 2D geometries (when described in terms of geodesic distances), but rather like fractal spaces with Hausdorff dimension $d_{H}^{\mathrm{sp}}=4[24,25]$. By contrast, typical space-time surfaces contributing to the path integral of 2D Lorentzian quantum gravity can be viewed as two-dimensional, as shown in $[4,8,9]$.

The spatial slices at constant integer $t$ are obviously Euclidean in nature, but it is not immediately clear how they will behave, since they appear as part of a larger foliated space-time geometry, and are coupled to each other in a nontrivial way. We have tried to extract the Hausdorff dimension
$d_{H}^{\mathrm{sp}}$ of the spatial slices lying inside the spherical universe, using the geodesic distance inherited from the 3D geometry, and employing techniques developed in the context of $2 \mathrm{D}$ dynamically triangulated Euclidean quantum gravity [26,27]. Unfortunately, the quality of our measurements is not very satisfactory, since the spatial volumes $N_{2}^{(s)}(t)$ of the individual slices are rather small (typically on the order of $1 k$ ).

One can obtain better data by using simulations with small $T$ (so that $T<T_{u}$ and no universe can form), but it is not entirely clear whether this will leave the spatial Hausdorff dimension unchanged. Our measurements for small $T$ point to a value around $d_{H}^{\mathrm{sp}}=3.4 \pm 0.4$ (the measurements for larger $T$ are compatible with this value, but their error bars are considerably larger). If our experience with the 2D Euclidean gravity simulations is anything to go by, this probably implies $d_{H}^{\mathrm{sp}}=4$, but so far this has to remain merely a conjecture. At any rate, these somewhat preliminary results highlight the fact that the detailed, microscopic geometry of the universe may be rather complicated, although its macroscopic properties resemble that of a semiclassical object.

Attempts to measure the Hausdorff dimension $d_{H}$ of the entire space-time (as opposed to that of individual spatial slices) have not yet led to unambiguous results. One wants to confine the measurement to the spherical universe, where again one runs into difficulties because of its relatively small radius. In addition, one needs a dynamical definition of where the universe begins and ends (along the $t$ direction), and must make sure that the result is independent of the particular prescription adopted. From the limited data collected (using the geodesic link or dual link distance, in the sense in which this notion is usually defined in dynamical triangulations) we conclude that the Hausdorff dimension is most likely larger than three.

Another important result concerns the relation between the geometries of different $k_{0}$, in the phase where $k_{0}<k_{0}^{c}$. In the numerical simulations we have observed the following:

(i) The distributions as functions of $t$ can be made to coincide for different $k_{0}$ by rescaling the time, $t \rightarrow f_{\mathrm{ti}}\left(k_{0}\right) t$ or alternatively $a_{t} \rightarrow f_{\mathrm{ti}}\left(k_{0}\right) a_{t}$, where $a_{t}$ is the link length in time direction. This is illustrated by the $N_{2}^{(s+1 / 2)}-N_{2}^{(s+1 / 2)}$ correlator $C(\Delta)$, Fig. 13, where we show both the actual and the rescaled distributions. 

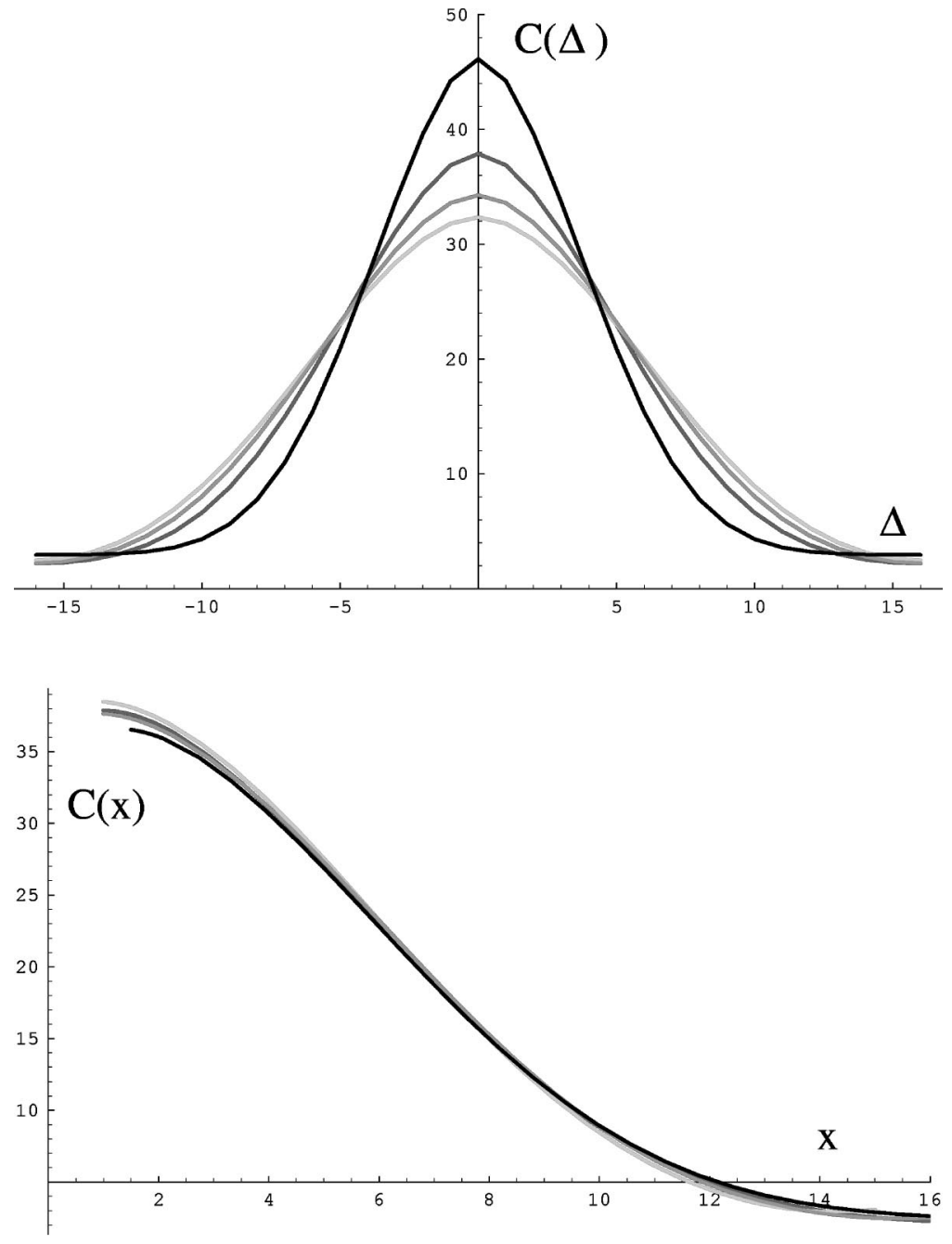

FIG. 13. The correlator $C(\Delta)$, Eq. (16) with $T=32$ and $N_{3}=16000$ measured for $k_{0}=3.0$, 4.0, 5.0, and 6.0 (lowest to highest peaks). The upper part shows the actual distribution, the lower one the rescaled distributions for positive $\Delta$ (it is symmetric in $\Delta$ ). The variable $x$ is a rescaled version of the time distance $\Delta$. (ii) The distributions measured in the spatial slices from inside the universe can be made to coincide for different $k_{0}$ by rescaling the spatial link distance $a_{s} \rightarrow f_{\mathrm{sp}}\left(k_{0}\right) a_{s}$, where $a_{s}$ is the length of the spatial links. This is illustrated in Fig. 14 for the distributions of 2D volumes $S(l)$ of spatial spherical shells of (link) radius $l$, measured for various values of $k_{0}$. (The shell volume $S(l)$ is obtained by counting the number of vertices separated from a given vertex $v$ by a minimal link distance $l$. Note that this spherical shell is precisely what is measured to determine the Hausdorff dimension $d_{H}^{\mathrm{sp}}$ of the spatial slices.)

(iii) Within the numerical accuracy we find that $f_{\mathrm{ti}}\left(k_{0}\right)$ $=f_{\text {sp }}\left(k_{0}\right)$. In fact, the rescaling of the correlator $C(\Delta)$ (Fig. 13) was obtained by simply using the values $f_{\mathrm{sp}}\left(k_{0}\right)$ (see Table I) determined from the fit $S(l)$ [rather than by finding the best value for $\left.f_{\mathrm{ti}}\left(k_{0}\right)\right]$.

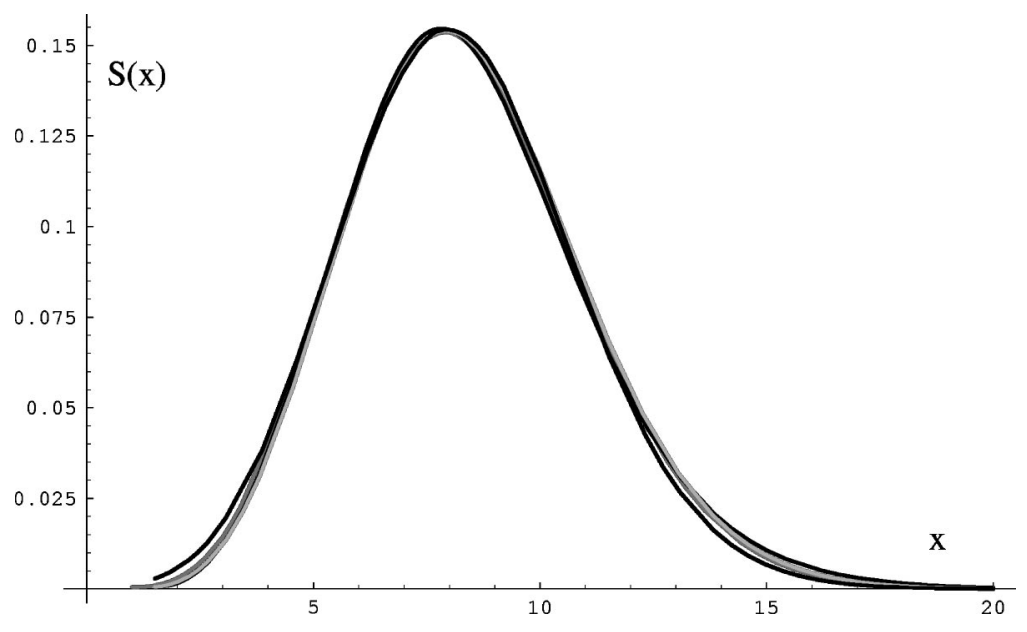

FIG. 14. The $2 \mathrm{~d}$ volume $S(x)$ of spatial spherical shells, measured only on slices inside the spherical universe, for various values of $k_{0}$ and rescaled. The variable $x$ is a rescaled version of the radius $l$. 
TABLE I. The spatial scaling factor $f_{\mathrm{sp}}\left(k_{0}\right)$, extracted from the distributions $S(l)$ for various values of $k_{0}$.

\begin{tabular}{cc}
\hline \hline$k_{0}$ & $f_{\mathrm{sp}}\left(k_{0}\right)$ \\
\hline 3.0 & $0.84 \pm .02$ \\
4.0 & $0.91 \pm .02$ \\
5.0 & 1.00 \\
6.0 & $1.23 \pm .03$ \\
\hline \hline
\end{tabular}

On the basis of these correlator measurements we conjecture that the value of the bare inverse gravitational coupling $\left.k_{0} \in\right] \widetilde{k}_{0}^{c}, k_{0}^{c}[$ merely sets the overall length scale of the universe, and otherwise does not affect the physics of the model.

We should point out that the average total integrated curvature is not independent of $k_{0}$. Subtracting the cosmological term from the action Eq. (A11), one finds

$$
\begin{aligned}
\int d^{3} x \sqrt{\operatorname{det} g} R & \rightarrow \pi a N_{3}\left(\tau\left(k_{0}\right)+(12 \kappa-5)-8 \frac{T}{N_{3}}\right) \\
& \sim \tau\left(k_{0}\right)-0.298-8 \frac{T}{N_{3}}
\end{aligned}
$$

where the parameter $\tau$ [defined in Eq. (10)] now depends dynamically on $k_{0}$ through the ensemble average. Comparing it with our measured curve for $\tau$ in Fig. 7, one can read off that the total curvature vanishes around $k_{0} \approx 5.0$. For smaller $k_{0}$, it becomes negative and for larger $k_{0}$ positive. Nevertheless, in line with our conjecture above we expect the curvature-curvature correlators to follow the pattern of the already measured correlators (i.e., to observe a simple $k_{0}$-dependent scaling behavior), but this remains to be verified.

\section{SUMMARY AND DISCUSSION}

In this paper, we have analyzed the phase structure of the discretized model of three-dimensional Lorentzian gravity defined in [5] with the help of computer simulations. The phase diagram, Fig. 15, should be compared with that of the Euclidean theory, depicted in Fig. 16. Although the overall phase structure is similar, with a first-order transition at some

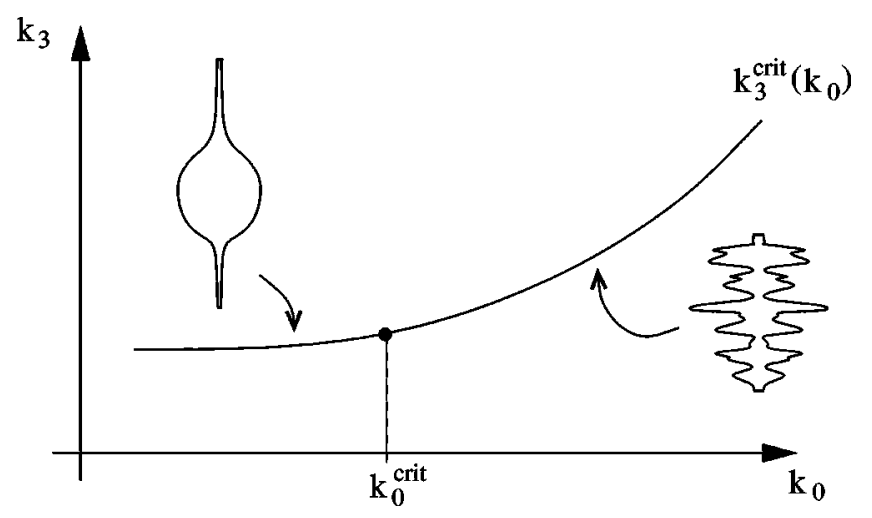

FIG. 15. The phase diagram of Lorentzian dynamical triangulations in three dimensions.

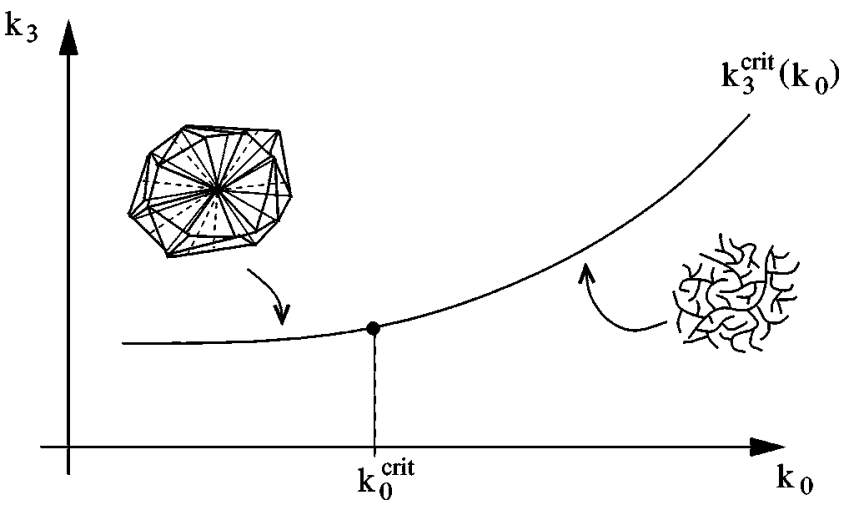

FIG. 16. The phase diagram of Euclidean dynamical triangulations in three dimensions.

intermediate value $k_{0}^{c}$, the quantum geometries of the phases on either side of the transition are very different in both cases, as indicated in the drawings.

In the Euclidean case [16], one finds a "crumpled" phase at small $k_{0}$, dominated by configurations of very large Hausdorff dimension $d \approx \infty$ (these are simplicial manifolds where roughly speaking any two vertices are a minimal distance apart). Above the first-order transition at $k_{0}^{c}$, the system is in a branched-polymer phase of highly branched geometries (with a fractal dimension $d_{H}=2$ ). Unfortunately, neither of these phases seems to have a ground state that resembles an extended geometry of dimension $d \geqslant 3$.

Another approach to Euclidean gravity was advocated in [28] within the quantum Regge calculus program. The phase structure found in the numerical simulations of this model resembles those of Figs. 15 and 16 at least superficially in exhibiting a "rough phase" for small and a "smooth phase" for large Newton's constant. However, this model is inequivalent to the dynamically triangulated models we have been discussing, since in the Regge approach these two phases seem to be separated by a second-order phase transition and associated divergent curvature fluctuations, indicating the presence of propagating field degrees of freedom (cf. our general discussion in Sec. II). How this can be related to the topological character of 3D quantum gravity manifest in canonical treatments of the theory is unclear.

The situation in Lorentzian dynamically triangulated gravity is completely different. Although we find a weak "remnant" of the Euclidean degeneracy for $k_{0}>k_{0}^{c}$, where space-time decouples into a sequence of uncorrelated twodimensional slices, there is a whole phase below $k_{0}^{c}$ where the geometry is extended, with macroscopic scaling properties characteric of a three-dimensional universe. Quite remarkably, this is an example in three dimensions of the emergence of a well-defined ground state of geometry in a nonperturbative state sum for gravity. In a continuum language, this is the ground state of an effective action, where entropy contributions (in other words, the measure) play a crucial role. Apparently in our model these contributions are such that they outbalance potential conformal divergences coming from the Wick-rotated action (otherwise a welldefined ground state could not exist). From the evidence gathered so far, the physics in this extended phase is inde- 
pendent of the precise value of the bare gravitational coupling $k_{0}$. In the correlation functions we have measured, $k_{0}$ merely serves to set an effective overall length scale.

As argued in Sec. II, these findings strongly favor a situation where the gravitational coupling is not renormalized, and no fine tuning of $k_{0}$ is needed to approach the continuum limit. This limit coincides automatically with the infinite (lattice) volume limit, which we obtained by fine tuning the cosmological coupling constant. Continuum physics is then extracted by taking the limit as $N_{3} \rightarrow \infty$ and $a \rightarrow 0$, while keeping the three volume $V_{\text {cont }}:=N_{3} a^{3}$ constant. In this setting, no genuine field degree of freedom is present since there is no divergent correlation length associated with fine tuning $k_{0}$ to the critical point of a second-order phase transition.

As a result of our investigations, we have good reasons to believe that 3D Lorentzian quantum gravity, as defined through our discrete, dynamically triangulated model, exists as a continuum theory. Since so far this theory is not given in an explicit analytical form, the question arises of how to make contact with already existing quantizations of threedimensional gravity.

At least in spirit, our formulation is related to canonical approaches using the trace of the extrinsic curvature as a time variable, the so-called "York time," with a conjugate Hamiltonian determining the time evolution of the system $[29,13]$. However, this approach only works for genus $g$ $\geqslant 1$, and the only case where the canonical quantum theory and the Hamiltonian operator are reasonably explicit is $g$ $=1$, where the spatial slices have torus topology. We are not aware of any quantum observables that have been calculated in the case of spherical slices which we could try to compare to. (For $S^{2}$ slices, there are no noncontractible holonomies and the reduced phase space is zero dimensional.) We could in principle repeat the simulations for toroidal spatial slices, although the finite-size effects will be larger for this more complicated topology (and for $T=32,64$, our spatial slices are rather small).

Similarly, it is in principle straightforward to enlarge the Lorentzian model to include coupling to matter fields. This has already been done in two-dimensional Lorentzian quantum gravity, with a clear motivation in mind, namely, to understand the status of the $c=1$ barrier in general 2D gravity models. We showed that this disease of 2D Euclidean quantum gravity can be avoided by working with Lorentzian geometries [9].

It would provide a strong incentive for considering either of these generalizations if there were definite predictions from continuum formulations of 3D quantum gravity with and without matter for observables measurable in the computer simulations (for example, correlation functions of the type we have been studying).

A next important step in our analysis of 3D Lorentzian quantum gravity will be the derivation of the explicit form of the Hamiltonian in the continuum limit. We can in principle obtain the matrix elements of the transfer matrix $\hat{T}$ between two successive triangulated two geometries $g_{i}$, as the solu- tion to a combinatorial problem [22]. Expanding the matrix elements according to

$$
\left\langle g_{2}|\hat{T}| g_{1}\right\rangle=\left\langle g_{2}\left|e^{-a \hat{H}}\right| g_{1}\right\rangle=\left\langle g_{2}\left|\left(1-a \hat{H}+O\left(a^{2}\right)\right)\right| g_{1}\right\rangle,
$$

one can extract the Hamiltonian operator $\hat{H}$. A similar approach was successful in 2D Lorentzian gravity, where the regularized transfer matrix could be calculated, and its continuum limit taken in a straightforward way. ${ }^{7}$ The resulting Hamiltonian agreed with the one obtained by continuum formal manipulations in the proper-time gauge [30], showing that the educated guesses made in this paper were justified.

This calculation can be generalized to our 3D Lorentzian gravity model, but the matrix-model methods will probably only work in the case of a spherical spatial topology. A direct comparison with canonical quantum gravity would then require a canonical continuum quantization in the proper-time gauge, with spatial topology $S^{2}$.

Let us conclude by pointing out an interesting consequence of our arguments that would follow if the second critical point $\widetilde{k}_{0}^{c}$ (whose value we did not measure) were negative. In this case, the theory with bare coupling constant $k_{0}=0$ would lie inside the extended phase. This implies that we could start from a discretized gravity action with the cosmological term alone, and still obtain the same continuum theory. This may seem to be a radical suggestion, because the classical theory of the action

$$
S=\Lambda \int d^{3} x \sqrt{\operatorname{det} g(x)}
$$

is trivial (it does not contain any time derivatives). However, there is no logical contradiction, since further nontrivial contributions to the (effective) action can be generated through the nonperturbative evaluation of the path integral. In fact, 2D Euclidean quantum gravity provides a good illustration of this mechanism. There the action is given by Eq. (20), but substituting $d^{3} x \rightarrow d^{2} x$. Nevertheless, the effective quantum theory in conformal gauge is described by the highly nontrivial quantum Liouville theory.

What have we learned from our exploration about our ultimate goal, the construction of quantum gravity in four dimensions? We invented the discrete Lorentzian models in the hope that they may lead to a better description of physical four-dimensional space-time, which after all has a Lorentzian signature. We also conjectured in [5] that in the continuum limit the causality constraints imposed on each geometry in the state sum may lead to a suppression of the degenerate phases of highly fractal geometry found in the Euclidean models for $d \geqslant 3$. From the evidence presented in this work, this is indeed what happens in three dimensions. Moreover, we saw the emergence of a ground state of extended three-dimensional geometry in the Lorentzian case. As already observed in $d=2$, also in three dimensions the

\footnotetext{
${ }^{7}$ The formula for the Hamiltonian in [4] contains a typo; see [17] for the correct expression.
} 
Euclidean and Lorentzian models correspond to completely different continuum theories, reiterating that these two "sectors" of the gravitational quantum theory are not related by a simple analytic continuation in time [4,31].

We are very encouraged by these results, since they also indicate that in $d=4$ completely different geometries will dominate the Wick-rotated path integral, compared with the Euclidean theory. The physics that the four-dimensional model should describe, if it were to lead to a nonperturbative theory of quantum gravity, must of course be very different from that found in two and three dimensions. In particular, the critical behavior of the regularized theory should reflect the presence of physical, propagating field degrees of freedom. In the context of the statistical models we are considering, the simplest realization would be in terms of a secondorder phase transition. This possibility is apparently not realized in the dynamically triangulated Euclidean gravity models. However, there is by now plenty of evidence that the Lorentzian model for quantum gravity defined in $[5,6]$ is sufficiently different to make it a new, promising candidate for a nontrivial nonperturbative theory of quantum gravity in four dimensions.

\section{ACKNOWLEDGMENTS}

All the authors acknowledge support by the EU network on "Discrete Random Geometry," Grant No. HPRN-CT1999-00161, and by ESF network No. 82 on "Geometry and Disorder." In addition, J.A. and J.J. were supported by "MaPhySto," the Center of Mathematical Physics and Stochastics, financed by the National Danish Research Foundation, and J.J. by KBN Grant Nos. 2P03B 01917 and 99814.

\section{APPENDIX A}

In this appendix we collect some formulas for dynamically triangulated three geometries, which were used in deriving various forms of the discrete Einstein action in the main text. We will work in the Euclidean sector of the theory, and for simplicity choose all tetrahedra to be equilateral (that is, $\alpha=-1$ and $l_{\text {space }}=l_{\text {time }}=a>0$ ). The curvature of a $3 \mathrm{D}$ piecewise linear manifold is concentrated at its links. The contribution to the total curvature associated with each link $l$ is given by the link length $a$ times the deficit angle

$$
\delta_{l}=2 \pi-\sum_{\sigma_{i} \supset l} \theta_{i}
$$

where the sum is taken over all tetrahedra $\sigma_{i}, i$ $=1, \ldots, o(l)$, sharing the link $l$, and $\theta_{i}$ is the dihedral angle associated with the $i$ th tetrahedron. For an equilateral three complex, all dihedral angles are identical

$$
\theta=\arccos \frac{1}{3} \equiv \kappa \pi,
$$

and the curvature term of the Einstein action becomes

$$
\frac{1}{2} \int d^{3} x \sqrt{\operatorname{det} g(x)} R(x) \rightarrow \sum_{l} a \delta_{l}=2 \pi a\left(N_{1}-3 \kappa N_{3}\right),
$$

where for a closed three-dimensional triangulation we have used

$$
\sum_{l} 1=N_{1}, \quad \sum_{l} o(l)=6 N_{3} .
$$

Taking into account that the three volume of an equilateral tetrahedron is given by $a^{3} / 6 \sqrt{2}$, we obtain the discretized form of the Euclidean Einstein action [5]

$$
S_{E}=-\frac{a}{4 G}\left(N_{1}-3 \kappa N_{3}\right)+\frac{a^{3} \Lambda}{48 \sqrt{2} \pi G} N_{3},
$$

where in a slight abuse of language we continue to use $G$ and $\Lambda$ to denote the bare gravitational and cosmological couplings. We can substitute $N_{1}$ by the number $N_{0}$ of vertices, using the identity $N_{1}=N_{3}+N_{0}$, which can be derived from the vanishing of the Euler number for any closed 3D manifold

$$
\chi=N_{0}-N_{1}+N_{2}-N_{3}=0,
$$

together with the relation $N_{2}=2 N_{3}$ (any triangle is shared by two tetrahedra and any tetrahedron has four triangles). Substituting this into Eq. (A4), we obtain the action used in Sec. I

$$
S_{E}=-k_{0} N_{0}+k_{3} N_{3},
$$

with the coupling constants given by

$$
k_{0}=\frac{a}{4 G}, \quad k_{3}=\frac{a^{3} \Lambda}{48 \sqrt{2} \pi G}+\frac{a}{4 G}(3 \kappa-1) .
$$

In the numerical investigation of Sec. III we discussed the dependence of the action on the total number $N_{22}$ of $(2,2)$ tetrahedra. This can be made explicit by rewriting $N_{0}$ as a function of $N_{3}$ and $N_{22}$. For periodic boundary conditions in the $t$ direction, the total numbers of $(3,1)$ and $(1,3)$ tetrahedra are the same, and we have

$$
N_{13}+N_{31}=\frac{1}{2}\left(N_{3}-N_{22}\right) \text {. }
$$

Next, we need some identities for the spatial slices at constant integer $t$. Because the slices are topologically twodimensional spheres, the number of vertices in a slice is

$$
N_{0}(t)=\frac{1}{2} N_{31}(t)+2=\frac{1}{2} N_{13}(t-1)+2 .
$$

Summing this equation over all $t$ and using Eq. (A8) yields

$$
N_{0}=\sum_{t=1}^{T} N_{0}(t)=2 T+\frac{1}{4}\left(N_{3}-N_{22}\right),
$$

and therefore

$$
S_{E}=\frac{k_{0}}{4} N_{22}+\left(k_{3}-\frac{k_{0}}{4}\right) N_{3}-2 K_{0} T,
$$




\section{APPENDIX B}

As discussed in Sec. III, one can describe the 3D Lorentzian geometries in terms of dual graphs, naturally associated with each plane of constant half-integer $t$. They decompose into two cubic graphs of different color (whose trivalent vertices correspond to the $(1,3)$ and $(3,1)$ tetrahedra of the original triangulation, and which may be thought of as the in and out-states of the transfer matrix. Gray and black lines cross at four-valent vertices, corresponding to the $(2,2)$ tetrahedra of the original lattice.

The planarity of this structure (i.e., the fact that the subgraphs have topology $S^{2}$ ) is easily implemented in the program by representing the one-dimensional lines of the gray and black graphs as double lines with opposite orientation, as one can do in the large- $n$ matrix model Eq. (9). In this way one obtains closed loops of oriented colored (single) lines which are dual to the vertices at times $t$ and $t+1$ of the original lattice. The 2D spherical surface may thus be thought of as being covered by (either gray or black) patches enclosed inside the loops.

In the numerical simulations we take care that the triangulations are 3D simplicial manifolds to start with, and we accept only Monte Carlo moves that preserve this property. In terms of the original triangulation, this means that we only allow the creation of configurations where any two vertices can be shared by at most one link, any three vertices can be shared by at most one triangle, and any four vertices by at most one tetrahedron.

In terms of the dual graphs, this implies two types of restrictions, the first of which have a transparent interpretation in the matrix model: they constrain the individual trivalent graphs to have neither tadpoles nor self-energy subdiagrams. This ensures that they are regular 2D simplicial manifolds with spherical topology. The remaining constraints restrict the ways in which the two colored graphs are allowed to intersect each other. Requiring the absence of double links between pairs of vertices that are time-like separated on the original lattice implies that the intersection of any pair of gray and black domains enclosed by gray and black loops cannot be multiply connected. Similarly the absence of double triangles from the original simplicial configuration means that the (one-dimensional) intersection of a double line of one color with a given domain inside a loop of the opposite color must be either empty or simply connected.

An important consequence of these constraints is that the number $N_{22}(t)$ of dual four-valent vertices is constrained both from below and above in terms of $N_{13}(t)$ and $N_{31}(t)$. This does not happen in the matrix model Eq. (9), where these numbers are completely independent. It is possible that some of the regularity conditions discussed here can be relaxed without affecting the universal properties, but for the 3D Lorentzian model this question has not yet been explored.

\section{APPENDIX C}

The numerical simulations presented in this paper were performed for system sizes of $4 \mathrm{k}, 8 \mathrm{k}, 16 \mathrm{k}, 32 \mathrm{k}$, and $64 \mathrm{k}$ tetrahedra, and for total proper times $T=16,32$, and 64. As usual, the standard unit was taken to be one sweep of the system, interpreted as $N_{3}$ attempted moves. Since the acceptance of moves is a function of $k_{0}$, in order that all moves were performed approximately the same number of times, we had to tune the number of attempted moves for each of the three types of moves appropriately. This technique has been applied successfully before in three- and fourdimensional simulations of Euclidean dynamical triangulations. We considered gravitational couplings in the range between $k_{0}=2.0$ and $k_{0}=7.0$. In this range the acceptance of move 1 is between $13.0 \%$ and $75 \%$, the acceptance of the moves 2 and 3 between $3 \%$ and $11 \%$, and that of moves 4 and 5 between $16 \%$ and $10 \%$. For even smaller $k_{0}$ the acceptance of the moves 2 and 3 decreases rapidly and it becomes very difficult with the present setup to change the geometry of the spatial intersections. A typical run corresponded to $10^{6}$ sweeps at a given value of $k_{0}$. For all measured quantities we found autocorrelation times below 100 sweeps, which was also the typical time between successive measurements.
[1] J. Ambjørn, in Fluctuating Geometries in Statistical Mechanics and Field Theory (Les Houches Summer School, Session LXII, 1994), edited by F. David, P. Ginsparg, and J. ZinnJustin (Elsevier, Amsterdam, 1996), pp. 77-193, hep-th/9411179.

[2] T. Regge, Nuovo Cimento A 19, 558 (1961).

[3] R. Loll, Living Rev. Relativ. 1, 13 (1998); http://www.livingreviews.org/Articles/Volume1/1998-131oll; gr-qc/9805049.

[4] J. Ambjørn and R. Loll, Nucl. Phys. B536, 407 (1998).

[5] J. Ambjørn, J. Jurkiewicz, and R. Loll, Phys. Rev. Lett. 85, 924 (2000).

[6] J. Ambjorn, J. Jurkiewicz, and R. Loll, Nucl. Phys. B (to be published), hep-th/0105267.

[7] J. Ambjørn, R. Loll, J. L. Nielsen, and J. Rolf, Chaos, Solitons Fractals 10, 177 (1999).
[8] J. Ambjørn, K. N. Anagnostopoulos, and R. Loll, Phys. Rev. D 60, 104035 (1999).

[9] J. Ambjørn, K. N. Anagnostopoulos, and R. Loll, Phys. Rev. D 61, 044010 (2000).

[10] J. Ambjørn, J. Jurkiewicz, and R. Loll, Nucl. Phys. B (Proc. Suppl.) 94, 689 (2001).

[11] R. Loll, Nucl. Phys. B (Proc. Suppl.) 94, 96 (2001).

[12] R. Loll, J. Math. Phys. 36, 6494 (1995).

[13] S. Carlip, Quantum Gravity in 2+1 Dimensions (Cambridge University Press, Cambridge, England, 1998).

[14] R. Sorkin, Phys. Rev. D 12, 385 (1975); 23, 565(E) (1981).

[15] F. David, in Gravitation and Quantizations (Les Houches Summer School, Session LVII, 1992), edited by J. Zinn-Justin and B. Julia (Elsevier, Amsterdam, 1995), pp. 679-750.

[16] J. Ambjørn, D. V. Boulatov, A. Krzywicki, and S. Varsted, Phys. Lett. B 276, 432 (1992); J. Ambjørn and J. Jurkiewicz, 
ibid. 278, 42 (1992).

[17] J. Ambjorn, J. Jurkiewicz, and R. Loll, in M-Theory and Quantum Geometry, edited by L. Thorlacius and T. Jonsson, NATO Science Series (Kluwer Academic, Dordrecht, 2000), pp. 382449, hep-th/0001124.

[18] V. Moncrief, J. Math. Phys. 31, 2978 (1990).

[19] H. Kawai and M. Ninomiya, Nucl. Phys. B336, 115 (1990).

[20] H. Kawai, Y. Kitazawa, and M. Ninomiya, Nucl. Phys. B404, 684 (1993).

[21] H. Kawai, Y. Kitazawa, and M. Ninomiya, Nucl. Phys. B467, 313 (1996).

[22] J. Ambjørn, J. Jurkiewicz, R. Loll, and G. Vernizzi, "Lorentzian 3d gravity with wormholes via matrix models," hep-th/0106082.

[23] A. Dasgupta and R. Loll, "A proper-time cure for the confor- mal sickness in quantum gravity," hep-th/0103186.

[24] H. Kawai, N. Kawamoto, T. Mogami, and Y. Watabiki, Phys. Lett. B 306, 19 (1993).

[25] J. Ambjørn and Y. Watabiki, Nucl. Phys. B445, 129 (1995).

[26] J. Ambjorn and J. Jurkiewicz, Nucl. Phys. B451, 643 (1995); J. Ambjørn, J. Jurkiewicz, and Y. Watabiki, ibid. B454, 313 (1995).

[27] S. Catterall, G. Thorleifsson, M. Bowick, and V. John, Phys. Lett. B 354, 58 (1995).

[28] H. W. Hamber and R. M. Williams, Phys. Rev. D 47, 510 (1993).

[29] V. Moncrief, J. Math. Phys. 30, 2907 (1989).

[30] R. Nakayama, Phys. Lett. B 325, 347 (1994).

[31] J. Ambjørn, J. Correia, C. Kristjansen, and R. Loll, Phys. Lett. B 475, 24 (2000). 